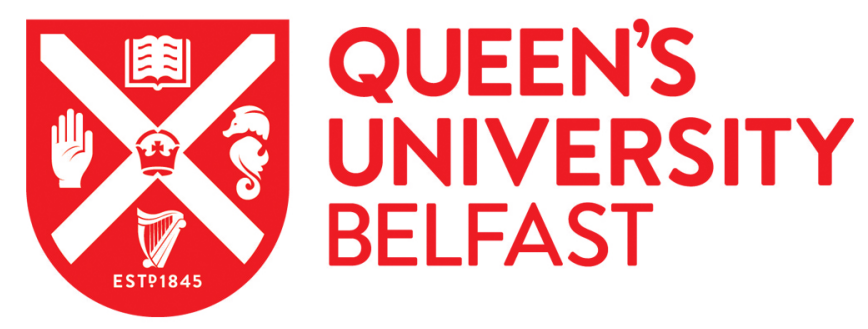

\title{
Robust fault estimation of a blade pitch and drivetrain system in wind turbine model
}

Hamideh Sedigh Ziyabari, S., Aliyari Shoorehdeli, M., \& Karimirad, M. (2020). Robust fault estimation of a blade pitch and drivetrain system in wind turbine model. Journal of Vibration and Control. https://doi.org/10.1177/1077546320926274

Published in:

Journal of Vibration and Control

Document Version:

Peer reviewed version

Queen's University Belfast - Research Portal:

Link to publication record in Queen's University Belfast Research Portal

Publisher rights

Copyright 2020 The Authors. This work is made available online in accordance with the publisher's policies. Please refer to any applicable terms of use of the publisher.

\section{General rights}

Copyright for the publications made accessible via the Queen's University Belfast Research Portal is retained by the author(s) and / or other copyright owners and it is a condition of accessing these publications that users recognise and abide by the legal requirements associated with these rights.

Take down policy

The Research Portal is Queen's institutional repository that provides access to Queen's research output. Every effort has been made to ensure that content in the Research Portal does not infringe any person's rights, or applicable UK laws. If you discover content in the Research Portal that you believe breaches copyright or violates any law, please contact openaccess@qub.ac.uk. 


\title{
Robust fault estimation of a blade pitch and drivetrain system in wind turbine model
}

\section{S.Hamideh Sedigh Ziyabari ${ }^{1}$ and Mahdi Aliyari Shoorehdeli ${ }^{2}$ and Madjid Karimirad ${ }^{3}$}

\begin{abstract}
In this paper, a novel robust fault estimation scheme to ensure efficient and reliable operation of Wind Turbines (WTs) has been presented. WTs are complex systems with large flexible structures that work under very turbulent and unpredictable environmental conditions for a variable electrical grid. The proposed observer-based estimation scheme consists of a set of possible faults affecting the dynamics, sensors, and actuators of WTs. First, the pitch and drivetrain system faults occur simultaneously with process and sensor disturbances that are called unknown input signals. Second, through a series of coordinate transformations, the faulty subsystem is decoupled from the rest of the system. The first subsystem is affected by unknown inputs and the second one is affected by faults. A Reduced-order Unknown Input Observer (RUIO) is designed to reconstruct states accurately while a Reduced-order Sliding Mode Observer (RSMO) is designed for the second subsystem such that it is robust against unknown inputs and faults. Moreover, the RUIO guarantees the asymptotic stability of the error dynamics using the Lyapunov theory method and completely removing unknown inputs; on the other hand, RSMO is designed to reconstruct faults for the faulty subsystem accurately. Until now, authors only focused on an unknown input signal in the dynamics of the system, especially in non-linear systems. The estimated fault will be adequate to accommodate the control loop, and sufficient conditions are developed to guarantee the stability of the state estimation error. In the next step, to figure the effectiveness of the proposed approach, a WT benchmark system model is considered with faults and unknown inputs scenarios. The simulation results will be used to validate the robustness of proposed algorithms under noise conditions, and the results show that the algorithm could classify faults robustly.
\end{abstract}

\section{Keywords}

Robust fault estimation, Pitch and drivetrain fault, Reduced-order unknown input observer, Reduced-order sliding mode observer, Orthogonal transformation, Wind turbine

\section{Introduction}

Nowadays renewable energies play a more sufficient role in power generation in the world. Energy production based on combustion of fossil fuels, such as coal or oil, has led to increasing temperature and greenhouse gasses. Wind turbines (WTs) have to compete with many other energy sources, and it is essential that they should be cost-effective, convenient and reliable. WTs are exposed to extremely variable and harsh conditions such as severe winds, lighting, arctic cold, hail, and snow; further, they need to meet different loads and produce stable energy. Marquez et al. (2012) explained for a 20-year old turbine, the operation, and maintenance costs of $750 \mathrm{Kw}$ turbines might be around $25-30 \%$ of the overall power generation cost or around $75-90 \%$ of the investment cost.

High mechanical stress on WTs because of extremely variable operating conditions and continuously changing, leads to more maintenance. Mainly, for WTs are located offshore. As a result, different approaches on fault diagnosis and control of WTs using a realistic WT benchmark Odgaard et al. (2009) and FAST (NREL) Jonkman et al. (2005) have been highlighted by different researchers in academia Wang et al. (2019); Witczak et al. (2016); Gao et al. (2016). The purpose of WT Fault Detection and Isolation (FDI) systems is to detect and locate degradation and failures in the operation of WT components as early as possible so that maintenance operations can be performed in due time. Therefore, the number of costly corrective maintenance actions can be reduced and consequently, the loss of wind power production due to maintenance operations is minimized. Most of the FDI algorithms for WTs were based on some form of signal analysis approach Qiao and Lu (2015). The signals used for fault diagnosis for WTs, mainly include vibration, acoustic emission (AE), strain, torque, temperature, lubrication oil parameters, electrical and supervisory control and data acquisition (SCADA) system signals. The signal processing approaches for fault diagnosis used typically includes classical time-domain analysis methods like statistical analysis Marquez et al.

\footnotetext{
${ }^{1}$ Research Laboratory for Fault Detection and Identification (FDI) Faculty of Electrical Engineering, K. N. Toosi University of Technology, Tehran, Iran

${ }^{2}$ Faculty of Electrical Engineering Department of Mechatronics Engineering, K. N. Toosi University of Technology, Tehran, Iran

${ }^{3}$ School of Natural and Built Environment, Queen's University Belfast, Northern Ireland, United Kingdom
}

\section{Corresponding author:}

Mahdi Aliyari Shoorehdeli, Department of Mechatronics Engineering, K. N. Toosi University of Technology, Tehran, Iran

Email: Aliyari@kntu.ac.ir 
(2012), classical frequency analysis methods like Fast Fourier Transform (FFT) Zhang et al. (2012), classical time-frequency analysis methods like wavelet transform Huang et al. (2008) and artificial intelligence (AI) methods Schlechtingen and Santos (2011). The fault mode and location can then be identified by a classification technique, like artificial neural networks (ANNs), support vector machines (SVMs) Laouti et al. (2011). Signal-based approaches such as neural networks and experts systems Sahin et al. (2012) are more suitable for information-poor systems. Compared with the signal processing methods for fault diagnosis for WTs, the model-based methods do not need high-resolution signals. The low-resolution, low-sampling-rate SCADA signals of a WT can be used effectively by model-based fault diagnosis. This is effective in installing additional sensors and data acquisition hardware to obtain high-resolution signals. In addition, the complex and costly computations in the signal-based methods to extract fault features from signals are unneeded in the model-based methods. The most commonly used approaches include Observer-based Chen et al. (2011), Parity space Ghaniee and Shoorehdeli (2011) and Parameter estimationbased Yin et al. (2012). In general, the parity space and parameter-based approaches are unsuitable for non-linear systems and will be unconsidered in this paper. The observerbased technique has received much attention to design a fault detection filter and is the base of this paper.

Model-based fault detection and isolation (FDI) is based on a residual signal that is generated by using the mathematical model of the system. In these methods, the objective is commonly obtained by comparing the system's measurements with the corresponding signals of its mathematical model. The difference between these signals called residuals is sensitive to any unknown input signals and faults. Since Model-based fault diagnosis methods have received a high sensitivity to corresponding mathematical models, it completely requires precise mathematical models which are not easy to derive. Any difference between the system and its models such as uncertainties, complexities, and unknown inputs cause seriously misleading alarms of faults; therefore, this problem has motivated to robust fault diagnosis schemes and many related studies have been done by other researchers Chen and Patton (2012); Nazir et al. (2017). Over the recent decades, many schemes have been achieved for FDI of non-linear systems with disturbance and fault together Lan and Patton (2017). On the other hand, it is challenging for a FDI scheme to provide the exact fault information, e.g. fault magnitude and shape. Compared with FDI strategies, fault identification or estimation technique can provide accurate fault information and it is able to react to the system in an Active Fault Tolerant Control (AFTC) and real-time decision Zhang and Jiang (2008); Azizi et al. (2019). Chen et al. (2011); Wei et al. (2010) proposed model-based FDI schemes employing a diagnostic observer for the pitch system and drivetrain faults on the benchmark model. Fault detection and isolation have been supported. Malik and Mishra (2015) a diagnostic technique for imbalance fault identification based on a probabilistic neural network was presented. Mathematical and statistical operations are performed on the measurements in signal processing fault diagnosis. Fault detection and isolation scheme in Dong and Verhaegen (2011) is a data-driven method to overcome the difficulty of modelling. Ghane et al. (2016); Feng and Liang (2014) demonstrated statistical change detection for a gearbox model of a wind turbine using frequency analysis. Fault detection, isolation, and accommodation to detect faults of the blade pitch systems have been considered in Cho et al. (2018). In this paper, to estimate the blade pitch angle, a Kalman filter is designed and a fault isolation scheme is proposed that could determine the fault type, location, magnitude. After that, the faulttolerant controller with a virtual sensor and shut-down mode controls WT to avoid unexpected external loads has been designed. Liu et al. (2017) focuses on robust fault estimation and tolerant control for T-S fuzzy model. A certain class of actuator and sensor faults considered generating an augmented system. Observability and some restricted conditions are limitations of this design. Simani and Castaldi (2019) proposed a non-linear relationship between measurements and faults of the offshore WT model with neural networks and fuzzy inference. It has been shown that due to the increased number of data in FWTs, implementing deep learning algorithms could be effective in the analysis of the operating condition. Fu et al. (2019) proposed a convolutional neural network $(\mathrm{CNN})$ to analyse the temperature data of gearbox bearing. Moreover, in another research, Wu et al. (2019) designed fault diagnosis with noisy measurements by denoising autoencoder (DAE) and Wang et al. (2016) proposed a deep autoencoder as an indicator of impending blade breakages. Data based methods need more sensors to analyse vibration, temperature, and other condition monitoring signals. Although the wind turbine industry has the interest to exploit effective condition monitoring methods, these methods suffer from high cost and complexity.

Fault estimation as one of the most important model-based fault diagnosis problems, which can also be perceived as the estimation of an unknown input has been done. In the literature, some of these fault estimation strategies usually attention, namely: system augmentation, especially the state vector by the new states Gao et al. (2016); Raoufi (2010), unbiased minimum-variance input and state estimation Gillijns and De Moor (2007), adaptive observers Azmi and Khosrowjerdi (2016); Guo et al. (2015), sliding mode observers Tan and Edwards (2003), high gain observers Veluvolu et al. (2011), $H_{\text {inf }}$ approach Raoufi et al. (2010) and unknown input observers Saoudi et al. (2015); Raoufi et al. (2010). The last two decades have witnessed enormous growth in fault diagnosis and to extend the previous approaches to non-linear systems. For robust fault estimation in non-linear systems, the observer-based approaches have gained many interest Raoufi and Marquezz (2010); Raoufi et al. (2010); Progovac et al. (2014); Guo et al. (2015). It should be emphasized that accurate fault estimation is a significant challenge for the non-linear system particularly in the presence of disturbances Mellucci et al. (2017) and definite faults Witczak (2014). Current solutions to robust fault estimation in a non-linear system are inadequate as well because there is not a distinct solution for non-linear observers Hassan (2002). In spite of its shortcomings, these methods have been widely employed to different kind of nonlinear systems. In Witczak et al. (2016); Raoufi et al. (2010) 
Lipschitz systems are considered that is a familiar form of uniform continuity for non-linear functions.

Disturbance attenuation by using optimization methods is generally more than using disturbance decomposition techniques. In contrast, disturbance decomposition can better relieve the adverse effects from the disturbances Witczak et al. (2016); Raoufi et al. (2010); Guo et al. (2015). A recent review of the literature on this area found that unknown input observers could completely attenuate unknown inputs like disturbances Raoufi and Marquezz (2010); Saoudi et al. (2015); Aldeen and Sharma (2008). In Raoufi and Marquezz (2010), both actuator and sensor fault simultaneously considers and augmented system is formed of a descriptor system. Although descriptor systems have been shown as conventional systems; it is difficult to solve their numerical problems, especially in industrial systems. Sliding mode observers (SMO) are robust against uncertainties and disturbance Edwards and Spurgeon (1998). SMO has been widely applied as an effective method to compensate for the uncertainty component in non-linear systems Yan and Edwards (2007). Indeed, it will be capable of preserving the error dynamics on a sliding surface in spite of the existence of uncertainty or fault. Furthermore, the exact estimation will exist and the equivalent output error signal that it is injected to observer structure will achieve an unwanted signal. In the fault estimation scheme, an unwanted signal is a fault and the equivalent output error signal will reach this after some transient time. Raoufi et al. (2010) a $H$ disturbance attenuation level proposed by the sliding mode observer design using LMIs optimization. The main contribution of this paper is using UIO for exact state estimation since UIO can exactly remove the dramatic effect of unknown inputs. Moreover, the equivalent output error signal in sliding mode structure will attain the unwanted signal. Ke Zhang and Cocquempot (2016) proposed a less conservative UIO design method by using a finite frequency range technique instead of an entirefrequency method. In Valibeygi et al. (2016) estimation of sensor faults in non-linear systems in the presence of process disturbance was considered. The unknown input observer for Lipschitz systems was applied for the fault diagnosis in Witczak et al. (2016). Ziyabari and Shoorehdeli (2017a) proposed a scheme that includes both component and sensor fault with the non-linear system that transferred to nonlinear T-S model to generate a residual signal. Moreover, Ziyabari and Shoorehdeli (2018) have designed the fault estimation scheme for the component fault. In addition, both studies have considered unknown inputs signals in a dynamic equation. Moreover, in Ziyabari and Shoorehdeli (2017b), the component fault was considered with the unknown input signal in state and output equations, but it was not included sensor and component fault estimation simultaneously. The essential contribution of this paper is a robust state and actuator fault estimator design procedure together. Two sources of uncertainty, unknown input, and exogenous external disturbance are present.

This paper is focused on the issue of robust fault estimation for a disrupted Wind Turbines (WT) subject to component and sensor faults and unknown inputs simultaneously via two reduced-order unknown input and sliding mode observers. It should also be pointed out that the proposed description of non-linearity is Lipschitz constraint. In this paper, the orthogonal transform has been improved, and the procedure for the component and sensor fault has been modified. This novelty does not lead to an increase the computation and there is not any restriction on time profile of faults, but faults should be norm bounded. This paper is motivated by the development of the Ziyabari and Shoorehdeli $(2017 b, 2018)$ by reforming the strategy of fault estimation to have disturbance in the measurements as well. The main contributions that are worth emphasizing are summarized in the following two aspects: (a) Through a coordinate transformation, the original system is decomposed into two separate subsystems. The first subsystem is affected only by faults and is free from unknown inputs; the second subsystem is affected by unknown inputs; (b) A UIO design is suggested to robustly estimate states to reconstruct faults in SMO. In addition, the analysis of the observers is formulated in terms of a series of LMIs that can be conveniently solved using LMI optimization technique such that a systematic calculation for observer gain matrices can be readily obtained using Matlab LMI toolbox. The effectiveness of the proposed method is demonstrated through its application to numerical simulation and compared to Ziyabari and Shoorehdeli (2017a). It will be indicated that these results clearly recommend the proposed approach for the purpose of AFTC. In contrast to the present works, the main contributions of this paper lie in the following aspects: (1) Robust fault estimation with two estimation mechanisms for unknown inputs in dynamic and measurement signals is designed; (2) To make a streamlined unknown input sliding mode observer, it is proved that the proposed controller guarantees that the closed-loop is asymptotically stable in the presence of faults and disturbances. (3) All possible faults (actuator, component, sensor) may happen in a real non-linear MIMO system simultaneously.

The rest of this paper is arranged as follows: First, provides a review of WT systems. The benchmark model and WTs faults and disturbances are presented to introduce the essential concepts of the system under study. Next section introduces the main ideas of the fault estimation scheme. First, reduced unknown input and sliding mode observers, second, fault estimation from previous observers has been done. Finally, with simulating the proposed scheme on WT benchmark system model, its ability for the industrial system has been demonstrated. A conclusion completes the paper.

\section{Wind turbine system description}

The WT considered in this paper was proposed in the benchmark described in Odgaard et al. (2009) that is a threeblade horizontal-axis turbine with a full converter coupling. The closed-loop system comprises five subsystems: aerodynamics, blade and pitch system, drivetrain, generator unit, controller and Figure 1 represents the relations between these subsystems faults and disturbance. The variables of WT are defined in Table 1 .

Exact descriptions of the sub-blocks, the mathematical models and the numerical values of the different parameters are given in the following sections. 


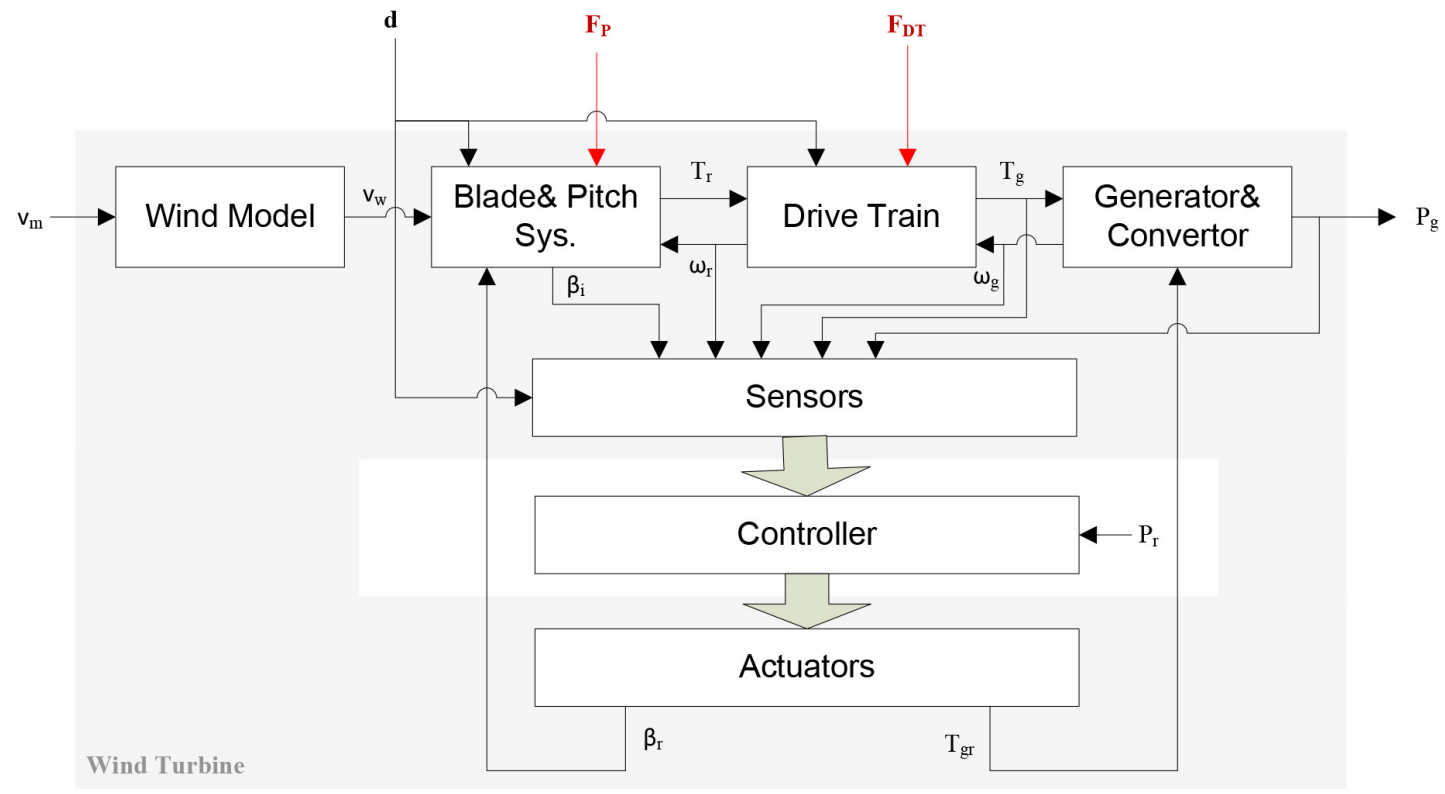

Figure 1. Interconnection of sub-models describing the characteristics of the wind turbine in Odgaard et al. (2009) benchmark.

Table 1. Wind model variables used in the benchmark model Odgaard et al. (2009)

\begin{tabular}{ll} 
Symbol & Description \\
\hline$\nu_{\omega}$ & wind speed on the turbine \\
$T_{\omega}$ & torque from wind on the blades \\
$T_{r}$ & rotor torque \\
$\omega_{r}$ & rotational speed of the rotor \\
$T_{g}$ & generator torque \\
$\omega_{g}$ & rotational speed of the generator \\
$\beta_{i, r}$ & reference to the pitch position \\
$\beta_{i}$ & the pitch position i \\
$F_{P}$ & fault in the pitch system \\
$F_{D T}$ & fault in the drivetrain system \\
$\tau_{g, r}$ & reference to generator torque \\
$P_{r}$ & power reference to the wind turbine \\
$P_{g}$ & power produced by the generator \\
\hline
\end{tabular}

\section{Wind turbine model}

The wind on the turbine blades forces the wind turbine rotor to spin around. Then, a rotating shaft converts the kinetic wind energy into mechanical energy. A generator, coupled to a converter, performs the conversion from mechanical energy to electrical energy. The system is operated by the wind speed that affects the aerodynamic properties of the wind turbine, together with the pitch angles of the blades and the speed of the rotor. An aerodynamic torque is transferred from the rotor to the generator through the drivetrain. Finally, the generator and converter provide electric power.

Aerodynamic model: A wind model that describes stochastic wind behaviour (speed variation and lateral effects of wind) is presented in Odgaard et al. (2009). It consists of the mean wind, a stochastic part, wind shear and tower shadow. The torque acting on the blades are modelled by aerodynamic $T_{r}(t)$. This model is a combination of the aerodynamic model and the wind and pitch model.

$$
T_{r}(t)=\sum_{i=1}^{3} \frac{\rho \pi R^{3} C_{q}\left(\lambda(t), \beta_{i}(t)\right) \nu_{w, i}^{2}(t)}{6}
$$

where $\rho$ is the air density, $R$ is the radius of the blades, $\nu_{w}$ is the wind speed and $C_{q}$ is the torque coefficient (the mapping used in this paper is in Odgaard et al. (2009)). This parameter characterizes the efficiency of the energy transfer from wind energy to mechanical energy, and it depends on the tip speed ratio $\lambda$ and the pitch angle $\beta$. This model for small differences between the pitch angles is valid.

Pitch system model: The hydraulic pitch system is modelled as a second-order system between the measured pitch angle $\beta_{i}$ and its reference $\beta_{i, r}$ :

$$
\ddot{\beta}_{i}=-2 \zeta_{i} \omega_{n, i} \dot{\beta}_{i}-\omega_{n, i}^{2} \beta_{i}+\omega_{n, i}^{2} \beta_{i, r}
$$

$\omega_{n, i}$ is the natural frequency and $\zeta_{i}$ is damping ratio with $i=1,2,3$. This is an individual blade pitch and the pitch angles $\beta_{i}, i=1,2,3$ are measured. $\beta_{i}$ is available for the wind turbine control system and for FDI and FTC schemes.

DriveTrain Model: The drivetrain model is an interconnection of a low-speed and a high-speed shaft by a transmission that has a gear ratio $N_{g}$ and an efficiency $\eta_{d t}$, combined with a torsion stiffness $K_{d t}$, and a torsion damping $B_{d t}$. The drivetrain transfers torque from the rotor to the generator. It includes a gearbox that increases the rotational speed from the low-speed rotor side to the high-speed generator side. Thus, the drivetrain can be described by the following three differential equations:

$$
\begin{gathered}
J_{r} \dot{\omega}_{r}=-\left(B_{d t}+B_{r}\right) \omega_{r}+\frac{B_{d t}}{N_{g}} \omega_{g}-K_{d t} \theta_{\delta}+T_{r} \\
J_{g} \dot{\omega}_{g}=\frac{\eta_{d t} B_{d t}}{N_{g}} \omega_{r}+\left(\frac{\eta_{d t} B_{d t}}{N_{g}^{2}}+B_{g}\right) \omega_{g}+ \\
\frac{\eta_{d t} K_{d t}}{N_{g}} \theta_{\delta}-T_{g} \\
\dot{\theta}_{\delta}=\omega_{r}-\frac{\omega_{g}}{N_{g}}
\end{gathered}
$$


where $J_{r}$ and $J_{g}$ low-speed shaft and high-speed shaft inertia, $B_{r}$ and $B_{g}$ friction coefficients, $\omega_{r}$ is the rotor speed, $\omega_{g}$ is the generator speed, $\theta_{\delta}$ is the torsion angle of the drivetrain, $T_{r}$ is the aerodynamic torque and $T_{g}$ is the generator torque. Both the rotor speed $\omega_{r}$ and the generator speed $\omega_{g}$ are measured.

Generator model: The generator torque $T_{g}$ is controlled by the reference $T_{g, r}$. The dynamics are approximated by a first-order model:

$$
\dot{T}_{g}=-\frac{T_{g}}{\tau_{g}}+\frac{T_{g, r}}{\tau_{g}}
$$

where $\tau_{g}$ is the time parameter of the generator subsystem. The power produced by the generator is given by:

$$
P_{g}=-\eta_{g} \omega_{g} T_{g}
$$

and $\eta_{g}$ is the efficiency of the generator and $\omega_{g}$ is the generator speed measurement. The values of the system parameters used in this paper have been extracted from Table 2.

Table 2. System parameters values

\begin{tabular}{lll}
\hline$R=57.5$ & $\rho=1.225$ & \\
$\omega_{n, 1}=11.11$ & $\omega_{n, 2}=5.73$ & $\omega_{n, 3}=3.42$ \\
$\zeta_{0}=0.6$ & $\zeta_{2}=0.45$ & $\zeta_{3}=0.9$ \\
$B_{d t}=775.49$ & $B_{r}=7.11$ & $B_{g}=45.6$ \\
$N_{g}=95$ & $K_{d t}=2.7 \mathrm{e} 9$ & $\eta_{d t}=0.97$ \\
$J_{g}=390$ & $J_{r}=55 \mathrm{e} 6$ & \\
$\tau_{g}=0.02$ & $\eta_{g}=0.98$ & \\
\hline
\end{tabular}

Finally, the general profile parameters of the wind turbine are presented in Table 3.

Table 3. General profile of wind turbine used in the benchmark model Odgaard et al. (2009)

\begin{tabular}{ll} 
Parameter & value \\
\hline Wind regime & stochastic wind \\
Rotor orientation & Clockwise rotation -Upwind \\
Type of wind turbine & horizontal-axis \\
Rated Power & $4.8 \mathrm{MW}$ \\
Rated wind speed & $12.5 \mathrm{~m} / \mathrm{s}$ \\
Number of blades & 3 \\
Converter coupling & full \\
Rated wind speed & $12.5 \mathrm{~m} / \mathrm{s}$ \\
Cut in wind speed & $3 \mathrm{~m} / \mathrm{s}$ \\
Cut out wind speed & $25 \mathrm{~m} / \mathrm{s}$
\end{tabular}

\section{Fault scenarios}

In this paper, sensor, actuator, and process faults in different parts of WT have been covered. Some of the faults are very serious and must be detected in a fast and safe condition. The rest is less critical, and the controller can accommodate these faults while staying in operation.

First, fault in the pitch position measurements is common sensor faults. The origin of these faults will be either electrical or mechanical and can result in either a fixed value or a changed gain factor on the measurements. The controller handles the pitch positions based on measurements, if it is not handled correctly, these faults will influence the system's performance.

Second, the deviations in rotor and generator speed measurements denote faults. Both the rotor and generator speed measurements are done using encoders and both electrical and mechanical failures are possible, which result in either a fixed value or a changed gain factor on the measurements. In case of a fixed value fault, the output of the encoder is not updated with new values. The gain factor fault is introduced when the encoder reads more marks on the rotating part than actually present, which can happen as dirt or other false alarm on the rotating part. Converter faults as a sample of actuator fault are denoted as an offset or in changing dynamics of the converter. This fault can be internal to the converter or because of electronics or an offset on the converter torque estimate.

Third, The pitch hydraulic system has the possibility of faults on all three blades. It can result in changing dynamics due to either a drop in pressure in the hydraulic supply system or high air content in the oil. The former represents faults like leakage in the hose, a blocked pump or similar others. There will always be some air content in the hydraulic oil used, the content level will vary, and it is not possible to control it well. Air is much more compressible than oil, so it alters the dynamics of the hydraulic actuator. Where the friction coefficient in the model changes slowly with time, the system fault will appear. This change will result in two correlated fault signals: $\omega_{r}$ and $\omega_{g}$. These changes evolve slowly in reality; however, it is expected to be extremely demanding in the benchmark simulator from a computational point of view to simulate such a fault realistically evolving over for months or even years. Consequently, in this benchmark model Odgaard et al. (2009), this fault is represented by a small change of the friction coefficient within a few seconds. The main point is that the change in the drivetrain friction is considerably slower than the system dynamics and the system sample rate. Typically, faults in wind turbine gearboxes are found using condition monitoring methods relying on additional sensors that measure accelerations and noise levels on the gearbox. It would be more cost-efficient if such faults could be detected and isolated using standard measurement in the wind turbine control system. A fault occurring in the pitch system can influence the closed-loop control system and the dynamics of a wind turbine. Faults of the blade pitch system are mainly categorized by the pitch sensor and actuator fault. The pitch sensor fault occurs by dust on encoder disc, miss-adjustment of the blade pitch bearing, beyond the acceptable range of temperature and humidity or improper calibration. These causes can result in the unbalanced rotation of the rotor from the sensor bias and fixed outputs from last measurements.

In this paper, the pitch system and in the drivetrain are considered for illustrating the proposed fault estimation approach. Combining results of the previous equation (2), together with sensor and actuator faults and disturbances, in the following set of the first-order differential equations

$$
\begin{aligned}
& \dot{x}_{p s}=A_{p s} x_{p s}+B_{p s} u_{p s}+E_{c} f_{a}(t)+E_{d} d(t) \\
& y_{p s}=C_{p s} x_{p s}+F_{d} d(t)+F_{c} f_{a}(t)
\end{aligned}
$$


where

$$
\begin{aligned}
& x_{p s}=\left[\begin{array}{llllll}
\ddot{\beta}_{1} & \dot{\beta}_{1} & \ddot{\beta}_{2} & \dot{\beta}_{2} & \ddot{\beta}_{3} & \dot{\beta}_{3}
\end{array}\right]^{T} \\
& u_{p s}=\left[\begin{array}{lll}
\beta_{1, r} & \beta_{2, r} & \beta_{3, r}
\end{array}\right]^{T} \\
& A_{p_{i}}=\left[\begin{array}{cr}
-2 \zeta_{i} \omega_{n, i} & -\omega_{n, i}^{2} \\
1 & 0
\end{array}\right], A_{p s}=\left[\begin{array}{lcr}
A_{p_{1}} & 0 & 0 \\
0 & A_{p_{2}} & 0 \\
0 & 0 & A_{p_{3}}
\end{array}\right] \\
& B_{p_{i}}=\left[\begin{array}{l}
1 \\
0
\end{array}\right], B_{p s}=\left[\begin{array}{ccr}
B_{p_{1}} & 0 & 0 \\
0 & B_{p_{2}} & 0 \\
0 & 0 & B_{p_{3}}
\end{array}\right] \\
& C_{p_{i}}=\left[\begin{array}{ll}
0 & \omega_{n, i}^{2}
\end{array}\right], C_{p s}=\left[\begin{array}{ccr}
C_{p_{1}} & 0 & 0 \\
0 & C_{p_{2}} & 0 \\
0 & 0 & C_{p_{3}}
\end{array}\right]
\end{aligned}
$$

for $i=1,2,3$ and the fault in the drivetrain consists of actuator and sensors with disturbances. Similarly to the pitch system case:

$$
\begin{aligned}
& \dot{x}_{d t}=A_{d t} x_{d t}+B_{d t} u_{d t}+E_{c} f_{a}(t)+E_{d} d(t) \\
& y_{d t}=C_{p s} x_{d t}+F_{d} d(t)+F_{c} f_{a}(t)
\end{aligned}
$$

where

$$
\begin{aligned}
& x_{d t}= {\left[\begin{array}{lll}
\omega_{r} & \omega_{g} & \theta_{\delta}
\end{array}\right]^{T}, \quad u_{d t}=\left[\begin{array}{ll}
T_{r} & T_{g}
\end{array}\right]^{T} } \\
& A_{d t}= {\left[\begin{array}{ccc}
-\frac{B_{d t}+B_{r}}{J_{r}} & \frac{B_{d t}}{N_{g} J_{r}} & \frac{-K_{d t}}{J_{r}} \\
\frac{\eta_{d t} B_{d t}}{N_{g} J_{g}} & \frac{\left(\frac{\eta_{d t} B_{d t}}{N_{g}^{2}}+B_{g}\right)}{J_{g}} & \frac{\eta_{d t} K_{d t}}{N_{g} J_{g}} \\
1 & -\frac{1}{N_{g}} & 0
\end{array}\right] } \\
& B_{d t}=\left[\begin{array}{lr}
\frac{1}{J_{r}} & 0 \\
0 & -\frac{1}{J_{g}} \\
0 & 0
\end{array}\right], C_{p s}=\left[\begin{array}{lll}
1 & 0 & 0 \\
0 & 1 & 0 \\
0 & 0 & 1
\end{array}\right]
\end{aligned}
$$

$d(t)$ and $f_{a}(t)$ are disturbance and fault for each subsystem and $E_{c}, F_{c}$ and $F_{d}$ are corresponding fault and disturbance distribution matrices with appropriate dimensions.

\section{System transformation}

Consider the following non-linear system:

$$
\begin{aligned}
& \dot{x}(t)=A x(t)+B u(t)+G f(x, u)+E_{d} d(t)+E_{c} f_{a}(t) \\
& y(t)=C x(t)+F_{d} d(t)+F_{c} f_{a}(t)
\end{aligned}
$$

where $x \in R^{n}, u \in R^{n_{u}}$ and $y \in R^{n_{y}}$ are the state vector, the input vector and the output vector, respectively; the known non-linear function $f(x, u)$ is Lipschitz with respect to $x$ uniformly for $u \in U$ ( $U$ is an admissible control set) with positive Lipschitz constant $\gamma_{0}$

$$
\left\|f\left(x_{1}, u\right)-f\left(x_{2}, u\right)\right\| \leq \gamma_{0}\left\|x_{1}-x_{2}\right\|
$$

and $E_{c}$ and $F_{c}$ are distribution matrices of component fault $f_{c} \in R^{n_{c}}$ and $f_{c}(t)$ is norm bounded with positive constants $\gamma_{1}$ as follows :

$$
\left\|f_{a}(t)\right\| \leq \gamma_{1}
$$

$d(t) \in R^{n_{d}}$ contains the uncertainty and disturbance, in the general case, it is commonly called unknown input signal. The number of measurements is more than the number of faults occur in the system $\left(n_{c}<n_{y}\right)$ and this assumption can be overcome by using more than one observer, each observer for a subset of the faults that satisfy this assumption. $A, B, G, E_{d}, E_{c}, C, F_{d}$ and $F_{c}$ are real constant known matrices of appropriate dimensions.
Lemma 1. For any matrices $X$ and $Y$ with appropriate dimensions, the following property holds for any positive scalar $\epsilon$ Alessandri (2004):

$$
X^{T} Y+Y^{T} X \leq \epsilon X^{T} X+\epsilon^{-1} Y^{T} Y
$$

Lemma 2. There exists a coordinate system in which the set $\left(E_{d}, E_{c}, F_{d}, F_{c}\right)$ has the following structure:

$$
\begin{aligned}
& \left(\left[\begin{array}{c}
e_{d} \\
0_{\left(n-n_{d}\right) \times n_{d}}
\end{array}\right], \quad\left[\begin{array}{c}
0_{n_{d} \times n_{c}} \\
e_{c} \\
f_{d} \\
0_{\left(n_{y}-n_{d}\right) \times n_{d}}
\end{array}\right], \quad\left[\begin{array}{c}
0_{n_{d} \times n_{c}} \\
f_{c}
\end{array}\right]\right)
\end{aligned}
$$

where $e_{d} \in R^{n_{d} \times n_{d}}, e_{c} \in R^{\left(n-n_{d}\right) \times n_{c}}, f_{d} \in R^{n_{d} \times n_{d}}, f_{c} \in$ $R^{\left(n_{y}-n_{c}\right) \times n_{d}}$ and $e_{d}, f_{d}$ should be full rank matrices.

\section{Proof:}

Without loss of generality, it can be assumed that there exists a QR transformation $T_{0}$ such that:

$$
T_{0} E_{d}=\left[\begin{array}{c}
e_{d} \\
0
\end{array}\right]
$$

where $e_{d} \in R^{n_{d} \times n_{d}}$. By using $T_{0}$, matrix $E_{c}$ has been following partitions:

$$
T_{0} E_{c}=\left[\begin{array}{l}
e_{1} \\
e_{c}
\end{array}\right]
$$

Now, introduce a non-singular transformation $T_{1}$ as

$$
T_{1}=\left[\begin{array}{lr}
I_{n_{d}} & -e_{1} e_{c}^{-1} \\
0_{\left(n-n_{d}\right) \times n_{d}} & I_{n-n_{d}}
\end{array}\right]
$$

then

$$
T_{1} T_{0} E_{d}=\left[\begin{array}{c}
e_{d} \\
0
\end{array}\right], \quad T_{1} T_{0} E_{c}=\left[\begin{array}{c}
0 \\
e_{c}
\end{array}\right]
$$

Also, it can be assumed that there exists a QR transformation $S_{0}$ such that:

$$
S_{0} F_{d}=\left[\begin{array}{c}
f_{d} \\
0
\end{array}\right]
$$

where $f_{d} \in R^{n_{d} \times n_{d}}$. By using $S_{0}$, matrix $F_{c}$ has been following partitions:

$$
T_{0} F_{c}=\left[\begin{array}{l}
f_{1} \\
f_{c}
\end{array}\right]
$$

Now, introduce a non-singular transformation $S_{1}$ as

$$
S_{1}=\left[\begin{array}{cc}
I_{n_{d}} & -f_{1} f_{c}^{-1} \\
0_{\left(n_{y}-n_{d}\right) \times n_{d}} & I_{\left(n_{y}-n_{d}\right)}
\end{array}\right]
$$

then

$$
S_{1} S_{0} F_{d}=\left[\begin{array}{c}
f_{d} \\
0
\end{array}\right], \quad S_{1} S_{0} F_{c}=\left[\begin{array}{c}
0 \\
f_{c}
\end{array}\right]
$$

Now $S_{1} S_{0} C T_{0}^{-1} T_{1}^{-1}$ partitions as

$$
\begin{gathered}
S_{1} S_{0} C T_{0}^{-1} T_{1}^{-1}=\left[\begin{array}{ll}
c_{1} & c_{2} \\
c_{3} & c_{4}
\end{array}\right] \\
T A T^{-1}=\left[\begin{array}{ll}
a_{1} & a_{2} \\
a_{3} & a_{4}
\end{array}\right]
\end{gathered}
$$


Then $T=T_{1} T_{0}, S=S_{1} S_{0}$.

Lipschitz systems are a very fundamental class and many non-linearities are locally Lipschitz. Also, Lipschitz nonlinear systems have received much attention in a different class of non-linear systems Valibeygi et al. (2016); Azmi and Khosrowjerdi (2016); Witczak et al. (2016) Trigonometric non-linearities are a suitable example for Lipschitz nonlinear that are common in robotics and non-linearities that are square or cubic. Relation (14) is not a conservative condition for fault estimation design because of having bounded signals to measure for fault diagnosis system is a healthy condition.

\section{Robust fault estimation architecture}

In this section, a robust fault estimation scheme will be introduced using the structure characteristics shown in the section "System transformation." Without loss of generality, the transformations are introduced in Lemma (2) as key to address the problem of robust fault estimation design which are $\bar{x}:=\left[x_{1}^{T}, x_{2}^{T}\right]^{T}=T x, \bar{y}:=\left[y_{1}^{T}, y_{2}^{T}\right]^{T}=S y$, the original system (12) can be rewritten

$$
\begin{aligned}
& \dot{x}_{1}=a_{1} x_{1}+a_{2} x_{2}+b_{1} u+g_{1} f\left(T^{-1} \bar{x}, u\right)+e_{d} d(t) \\
& \dot{x}_{2}=a_{3} x_{1}+a_{4} x_{2}+b_{2} u+g_{2} f\left(T^{-1} \bar{x}, u\right)+e_{c} f_{a}(t) \\
& y_{1}=c_{1} x_{1}+c_{2} x_{2}+f_{d} d(t) \\
& y_{2}=c_{3} x_{1}+c_{4} x_{2}+f_{c} f_{a}(t)
\end{aligned}
$$

where $x_{1} \in R^{n_{d}}, x_{2} \in R^{n-n_{d}}$ are partitioned transformed state of the original system (12). $g_{1}, b_{1}$ and $g_{2}, b_{2}$ are the first $n_{d}$ and the last $n-n_{d}$ components of transformed $G, B$ respectively. $e_{d}, e_{c}, c_{1}, c_{2}, c_{3}$ and $c_{4}$ are defined from Lemma (2). Thus, the faulty state space and output equations have been decoupled from the fault-free state space and output equations. Given the above preliminaries and assumptions, the objective of the subsequent part of this paper is to provide a reduced-order unknown input estimation strategy to attenuate unknown inputs and another RUIO to attenuate faults separately for the class of non-linear systems (12). The main advantage of the proposed approach is the fact that apart from estimating the sensor and component fault simultaneously, it is able to decouple the effect of unknown inputs in measurements and dynamics of the system within RUIOs.

\section{Reduced unknown input observer}

In this section, a theorem to design RUIO for the fault-free and faulty part of system (27) is separately proposed. The fault-free part will be rewritten as:

$$
\begin{aligned}
& \dot{x}_{1}=a_{1} x_{1}+\bar{b}_{1} \bar{u}_{1}+g_{1} f\left(T^{-1} \bar{x}, u\right)+e_{d} d(t) \\
& y_{1}=c_{1} x_{1}+\bar{d}_{1} \bar{u}_{1}+f_{d} d(t)
\end{aligned}
$$

where $\bar{u}_{1}=\left[x_{2}^{T} u^{T}\right]^{T}, \bar{b}_{1}=\left[a_{2}, b_{1}\right] \quad \bar{d}_{1}=\left[c_{2}, 0\right]$ and the RUIO is inferred as follows:

$$
\begin{aligned}
& \dot{z}_{1}=H_{1} z_{1}+J_{1} \bar{u}_{2}+W_{1}\left(y_{1}-\bar{d}_{1} \bar{u}\right)+Q_{1} g_{1} f\left(T^{-1} \hat{\bar{x}}, u\right) \\
& \hat{x}_{1}=z_{1}-R_{1}\left(y_{1}-\bar{d}_{1} \bar{u}\right)
\end{aligned}
$$

where $H_{1}, J_{1}, W_{1}, Q_{1}$ and $R_{1}$ for $i=1, \ldots, r$ are constant matrices with appropriate dimensions defined as:

$$
\begin{aligned}
& H_{1}=Q_{1} a_{1}-K_{1} c_{1}, \quad J_{1}=Q_{1} \bar{b}_{1}, \\
& Q_{1} e_{d}-K_{1} f_{d}=0, \quad R_{1} f_{d}=0 .
\end{aligned}
$$

Also, the faulty part is rewritten as follow:

$$
\begin{aligned}
& \dot{x}_{2}=a_{4} x_{2}+\bar{b}_{2} \bar{u}_{2}+g_{2} f\left(T^{-1} \bar{x}, u\right)+e_{c} f_{a}(t) \\
& y_{2}=c_{4} x_{2}+\bar{d}_{2} \bar{u}_{2}+f_{c} f_{a}(t),
\end{aligned}
$$

where $\bar{u}_{2}=\left[x_{1}^{T} u^{T}\right]^{T}, \bar{b}_{2}=\left[a_{3}, b_{2}\right] \bar{d}_{2}=\left[c_{3}, 0\right]$ and the UIO is inferred as follows:

$$
\begin{aligned}
& \dot{z}_{2}=H_{2} z_{2}+J_{2} \bar{u}_{2}+W_{2}\left(y_{2}-\bar{d}_{2} \bar{u}\right)+Q_{2} g_{2} f\left(T^{-1} \hat{\bar{x}}, u\right) \\
& \hat{x}_{2}=z_{2}-R_{2}\left(y_{2}-\bar{d}_{2} \bar{u}\right)
\end{aligned}
$$

where $H_{2}, J_{2}, W_{2}, Q_{2}$ and $R_{2}$ are constant matrices with appropriate dimensions defined as:

$$
\begin{array}{ll}
H_{2}=Q_{2} a_{4}-K_{2} c_{4}, & J_{2}=Q_{2} \bar{b}_{2}, \\
Q_{2} e_{c}-K_{2} f_{c}=0, & R_{2} f_{c}=0 .
\end{array}
$$

And $K_{1}, R_{1}$ and $K_{2}, R_{2}$ are independent variables and Proposition (1) will choose them. $z_{1} \in \Re^{n_{c}}$ and $z_{2} \in \Re^{n-n_{c}}$ are the observer states that generate exact estimated states $\hat{x}_{1}$ and $\hat{x}_{2}$. The necessary conditions to design observers are observability of $\left(a_{1}, c_{1}\right)$ and $\left(a_{4}, c_{4}\right)$, then the gains of the observer such that the state estimation error converges towards zero will be calculated. Sufficient conditions for each RUIO are given by (29)-(30) and (32)-(33), are described in the following Proposition.

Proposition 1. Given the non-linear system (28) with Lipschitz constant $\gamma_{0}$, consider UIO structure (29)-(30). The observer error dynamics is asymptotically stable such that $\epsilon>0, K_{1}, R_{1}$, and a positive-definite symmetric matrix $P_{1}$ will exist such that the following linear conditions hold:

$$
\left(\begin{array}{cr}
X & X_{12} \\
X_{12}^{T} & -\epsilon^{-1} I
\end{array}\right)<0
$$

where $X$ and $X_{12}$ are defined as:

$$
\begin{gathered}
X=\left(\left(P_{1}+\bar{R}_{1} c_{1}\right)-\bar{K} c_{1}\right)^{T}+\left(P_{1}+\bar{R}_{1} c_{1}\right) \\
-\bar{K} c_{1}+\gamma^{2} / \epsilon \\
X_{12}=P_{1} g_{1}+\bar{R}_{1} c_{1} g_{1}
\end{gathered}
$$

with $K_{1}=P_{1}^{-1} \bar{K}_{1}$ and $R_{1}=P_{2}^{-1} \bar{R}_{1}$.

Proof During the decoupling of non-linear system the transformed state error equation: $e_{1}=x_{1}-\hat{x}_{1}$

$$
e_{1}=\left(I+R_{1} c_{1}\right) x_{1}-z_{1}+R_{1} f_{d} f_{a}(t)
$$

The dynamics of state estimation error is given from (28) and (29) becomes:

$$
\begin{gathered}
\dot{e}_{1}=H_{1} e_{1}+\left(Q_{1} a_{1}-H_{1} Q_{1}-W_{1} c_{1}\right) x_{1} \\
+\left(Q_{1} \bar{b}_{1}-J_{1}\right) \bar{u}_{1}+Q_{1} g_{1} \tilde{f}+ \\
\left(Q_{1} e_{d}-W_{1} f_{d}-H_{1} R_{1} f_{d}\right) d(t)+R_{1} f_{d} \dot{d}(t)
\end{gathered}
$$

where $Q_{1}=\left(I+R_{1} c_{1}\right), K_{1}=W_{1}+H_{1} R_{1}$ and $\tilde{f}=f\left(T^{-1} \hat{\bar{x}}, u\right)-f\left(T^{-1} x, u\right)$. If the following conditions hold true $\forall i=1, \ldots, r$,

$$
\begin{aligned}
& H_{1}=Q_{1} a_{1}+K_{1} c_{1}, \quad J_{1}=Q \bar{B}_{2}, \\
& Q_{1} e_{d}-K_{1} f_{d}=0, \quad R_{1} f_{d}=0
\end{aligned}
$$

Then the equation of the observing error dynamics becomes:

$$
\dot{e_{2}}=H_{1} e_{1}+Q_{1} g_{1} \tilde{f}
$$


Let us consider the Lyapunov function $V\left(e_{1}(t)\right)=$ $e_{1}^{T}(t) P_{1} e_{1}(t)$, the derivative of the Lyapunov function is given by

$$
\dot{V}\left(e_{1}(t)\right)=e_{1}^{T}\left(H_{1}^{i^{T}} P_{1}+P_{1} H_{1}\right) e_{2}+2 e_{1}^{T} P_{1} Q_{1} g_{1} \tilde{f}
$$

Using the Lemma (1) and Lipschitz constrain (13),

$$
\begin{gathered}
\dot{V}\left(e_{1}(t)\right) \leq e_{1}^{T}\left(H_{1}^{i^{T}} P_{1}+P_{1} H+\epsilon P_{1} Q_{1}^{i^{T}} Q_{1} P_{1}+\right. \\
\left.\epsilon^{-1} \gamma_{0}^{2} I\right) e_{1}
\end{gathered}
$$

Stability condition for the estimation error yields to that the time derivative of the Lyapunov function should be negative define.

$$
H_{1}^{i^{T}} P_{1}+P_{1} H_{1}+\epsilon P_{1} Q_{1}^{T} Q_{1} P_{1}+\epsilon^{-1} \gamma_{0}^{2} I<-\alpha .
$$

By replacing $H_{1}$ and $Q_{1}$, then using the variable change $\bar{R}=P_{1} R_{1}$ and $\bar{K}_{1}=P_{1} K_{1}$, the last inequality (41) can be written as:

$$
\begin{gathered}
\left(\left(P_{1}+\bar{R} c_{1}\right)-\bar{K}_{1} c_{1}\right)^{T}+\left(P_{1}+\bar{R} c_{1}\right)-\bar{K}_{1} c_{1}+ \\
\epsilon P_{1} Q_{1} g_{1} g_{1}^{T} Q_{1}^{T} P_{1}+\epsilon^{-1} \gamma_{0}^{2} I
\end{gathered}
$$

Inequality (42) is given by using the Schur complement relation. Thus LMI (34) with parameters of (35) concludes. Since $\dot{V}$ in (41), a exponential function for upper bound of $e_{1}(t)$ exists as

$$
\left\|e_{1}(t)\right\| \leq M\left\|e_{1}(0)\right\| \exp \left(-\frac{\alpha t}{2}\right)
$$

where a choice is $M=\sqrt{\frac{\lambda_{\max }\left(P_{1}\right)}{\lambda_{\min }\left(P_{1}\right)}}$; as a result, there exits a dynamic system for upper bound of $M\left\|e_{1}(0)\right\| \exp \left(-\frac{\alpha t}{2}\right)$

$$
\dot{w}(t)=-\frac{1}{2} \alpha w(t)
$$

then, it is easy to see that $\left\|e_{1}(t)\right\| \leq w(t), \forall t \geq 0$.

A similar proof can be obtained for relations (31)-(33) for the fault free subsystem.

\section{Reduced sliding mode observer}

It is assumed that UIOs have been designed, a proposition to design SMO for faulty part of system (27) to estimate fault is proposed and the faulty part is (31). The RSMO is inferred as follows:

$$
\begin{aligned}
& \dot{\hat{x}}_{2}=a_{4} \hat{x}_{2}+\bar{b}_{2} \bar{u}_{2}+g_{2} f\left(T^{-1} \hat{\bar{x}}, u\right)+N\left(\hat{\bar{x}}_{2}-\hat{x}_{2}\right) \\
& +\nu(\hat{x}, \hat{\bar{x}}) \\
& \hat{y}_{2}=c_{4} \hat{x}_{2}+d_{2} \bar{u}_{2}
\end{aligned}
$$

where $\hat{\bar{x}}_{2}$ is estimated $\hat{x}_{2}$ from RUIO (32), $N$ is constant matrix with appropriate dimensions and the gain function $\nu($.$) is defined by$

$$
\nu(\hat{x}, \hat{\bar{x}}):=k(.) \frac{\hat{\bar{x}}_{2}-\hat{x}_{2}}{\left\|\hat{\bar{x}}_{2}-\hat{x}_{2}\right\|}
$$

where $k($.$) is a positive scalar function to be determined and$ a sliding surface

$$
S=\left\{t \in R^{+}: s(t)=0 \mid s(t)=\hat{\bar{x}}_{2}-\hat{x}_{2}\right\}
$$

is considered and $\hat{x}_{2}$ has affected by only fault signal; therefore, sufficient condition for guaranteeing the asymptotic convergence of fault estimation error and output estimation error system is described by the following Proposition.
Proposition 2. Given the non-linear system (31) with Lipschitz constant $\gamma_{0}$, consider RSMO structure (45)-(46). The observer error dynamics is asymptotically stable such that $N$ for $i=1, \ldots, r$ and a positive-definite symmetric matrix $P_{3}$ exist such that the following conditions hold:

$$
a_{4} P_{3}+P_{3} a_{4}^{T}-\bar{N}-\bar{N}^{T}<0
$$

with $N=P_{2}^{-1} \bar{N}$ and to derive the sliding surface (47) in finite time and remains on it if $k($.$) in (46) is chosen to satisfy$

$$
k(.) \geq\left(\left\|a_{3}\right\|+\left\|g_{2}\right\| \gamma_{0}\right) w(t)+\left\|e_{c}\right\| \gamma_{1}+\eta
$$

Proof. Let $e_{3}=\hat{\bar{x}}_{2}-\hat{x}_{2}$. Then from (45) and (32), the state estimation error is described by

$$
\dot{e}_{3}=\left(a_{4}-N\right) e_{3}-a_{3} e_{1}+g_{2} \tilde{f}+e_{c} f_{a}(t)-\nu
$$

let $V\left(e_{3}\right)=e_{3}^{T} P_{3} e_{3}$; therefore, the time derivative of $V$ along the trajectories of the system (50) is given by

$$
\begin{gathered}
\dot{V}\left(e_{3}\right)=e_{3}^{T}\left(\left(a_{4}-N\right) P_{3}+P_{3}\left(a_{4}-N\right)^{T}\right) e_{3}+ \\
2 e_{3}^{T} P_{3}\left(a_{3} e_{1}+g_{2} \tilde{f}+e_{c} f_{a}(t)-\nu\right)
\end{gathered}
$$

since by design symmetric negative definite first part of (51)

$$
\left(a_{4}-N\right) P_{3}+P_{3}\left(a_{4}-N\right)^{T}<0
$$

first condition (48) is concluded. By applying (13), (14) and (44) in (51)

$$
\begin{gathered}
\dot{V} \leq 2\left\|e_{3}\right\| \lambda_{\max }\left(P_{3}\right)\left(\left(\left\|a_{3}\right\|+\left\|g_{2}\right\| \gamma_{0}\right) w(t)\right. \\
+\left\|e_{c}\right\| \gamma_{1}-\nu
\end{gathered}
$$

thus second condition (49) is concluded.

\section{Fault estimation scheme}

It is assumed that the UIO and SMO are given, have been designed. In this part, the estimation of fault by using the SMO's output and output information is done. Basically the following theory is the result of two prior Propositions.

Theorem 1. Consider subsystem (31) exists and assume that the LMIs (34)-(48) are solvable and $k($.$) is chosen to$ satisfy (49),

$$
\hat{f}_{a}(t)=k(.) e_{c}^{-1} \frac{\hat{\bar{x}}_{2}-\hat{x}_{2}}{\left\|\hat{\bar{x}}_{2}-\hat{x}_{2}\right\|+\sigma_{1} \exp \left\{-\sigma_{2} t\right\}}
$$

Proof. In finite time the error dynamics (50) will be taken place on surface (47) and $\dot{e}_{3}=e_{3}=0$; therefore, during the sliding motion, the error dynamics for $e_{3}$ is given by

$$
\begin{gathered}
0=-a_{3} e_{1}+g_{2} \tilde{f}+e_{c} f_{a}(t)-\nu \\
-a_{3} e_{1}+g_{1} \tilde{f}+e_{c} f_{a}(t)=\nu
\end{gathered}
$$

where $\nu$ denotes discontinuous signal (46) must take on the average $\nu_{e q}$, the equivalent output error injection signal Edwards and Spurgeon (1998) to preserve the sliding motion. From Lipschitz constrain (13) and upper bound (44),

$$
\left\|-a_{3} e_{1}+g_{2} \tilde{f}\right\| \leq\left(\left\|a_{3}\right\|+\gamma_{0}\right)\left\|e_{1}\right\| \rightarrow 0(t \rightarrow 0)
$$




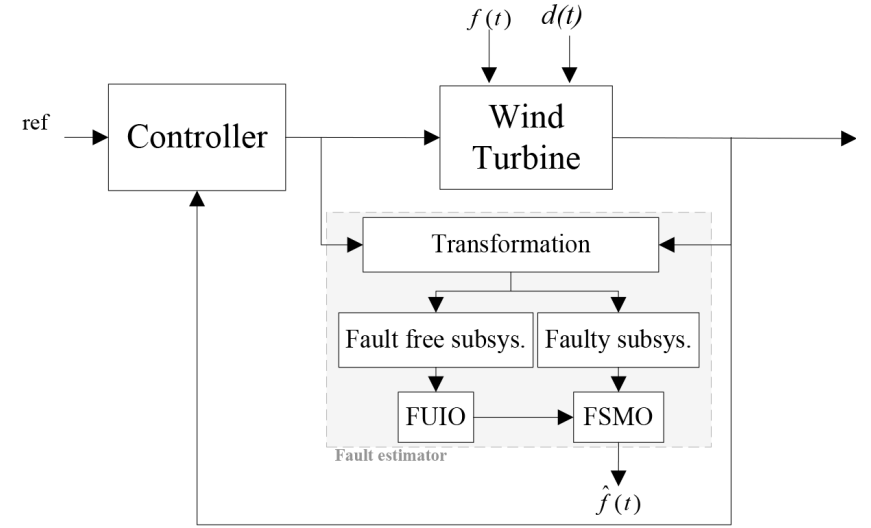

Figure 2. Suggested robust fault estimation scheme for Wind turbine with its interconnections.

thus estimation of fault $\hat{f}_{a}(t)$ is recovered by the equivalent output error injection signal $\nu_{e q}$.

$$
\nu_{e q} \simeq k(.) \frac{\hat{\bar{x}}_{2}-\hat{x}_{2}}{\left\|\hat{\bar{x}}_{2}-\hat{x}_{2}\right\|+\sigma_{1} \exp \left\{-\sigma_{2} t\right\}}
$$

where $\sigma_{1}$ and $\sigma_{2}$ are both positive constants such that

$$
\hat{f}_{a}(t) \simeq k(.) e_{c}^{-1} \frac{\hat{\bar{x}}_{2}-\hat{x}_{2}}{\left\|\hat{\bar{x}}_{2}-\hat{x}_{2}\right\|+\sigma_{1} \exp \left\{-\sigma_{2} t\right\}}
$$

Hence the proof completes.

Each element of $\nu_{e q}$ corresponds to the same fault, therefore we have accurate fault estimation. First set of RUIO can remove all disturbances or uncertainties and second set RSMO estimates exact fault; therefore, the estimated fault can be used for fault accommodation control. In the Figure 2 to make it clear the proposed fault estimation scheme, its subsystems and the signalling configuration has been shown Figure 1 next to Figure 2 shows a complete layout of faults, disturbances in wind turbine and the robust fault diagnosis scheme.

The disadvantage of the proposed approach may be offline computation and apply the result to real-life experiments; but, it will be an advantage when a real-time case is considered for less computational volume.

\section{Case study: Wind turbine}

In this section, simulation results of the proposed fault estimation scheme for the WT are presented. Simulations of the WT subjected to a stochastic wind speed under fault in pitch and drivetrain subsystems are conducted with respect to the fault-free case. The results prove the performance of the fault estimation technique under different fault scenarios. Wind speed time series with a mean speed of $12 \mathrm{~m} / \mathrm{s}$, and over $44000(\mathrm{sec})$ of simulation time is illustrated in Figure (3) with the wind elevation and four different wind speeds at hub height and three speeds at the three-blade tips, respectively, shown in Figure (4). In the following section, to test normal condition, performance and effectiveness of the controller presented. The wind profile is applied to the wind turbine without any sensor and actuator faults and disturbances. Then, in fault scenario sections, faults in the drivetrain and pitch subsystems are considered and the time increment is chosen $0.01(\mathrm{sec})$.

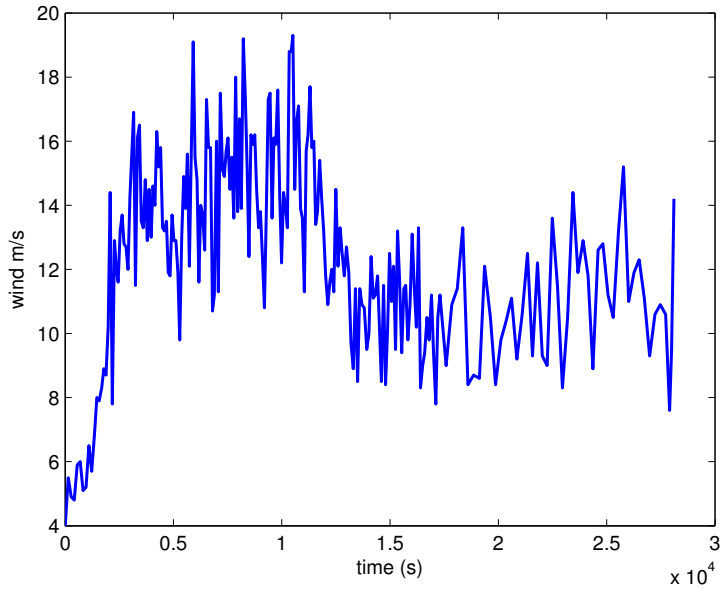

Figure 3. Wind speed time series with a mean speed of $12 \mathrm{~m} / \mathrm{s}$.

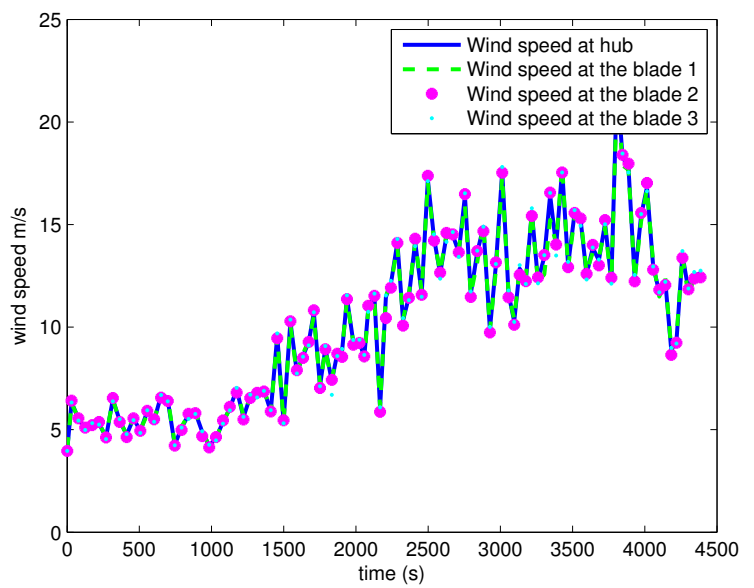

Figure 4. Wind speed time series at the hub (blue solid line), blade 1 (green dashed line), 2 (magenta asterisk) and 3 (cyan point) for WT.

\section{Fault-free case}

In this section, the wind turbine works in two specific regions of the graph are shown in Figure 5. In zone 1, the wind turbine will be awaiting higher wind speeds 0 $3 \mathrm{~m} / \mathrm{s}$. In zone 2 , the generated power of the wind turbine will be optimized and in zone 3, the wind turbine will be controlled to keep a constant power generation. In zone 4 , the wind turbine will be parked, preventing damage due to the high wind speed. Zone 2 is denoted the power optimization and zone 3 is denoted power reference and these are corresponded to control modes 1 and 2. In this paper, a simple control scheme Odgaard et al. (2009) has been used and the focus is on the fault estimation of the wind turbine. Figure 6 shows the control procedure for power and pitch-regulated wind turbine algorithm. In Figure $6, \omega_{\text {nom }}$ and $\omega_{\delta}$ are the nominal generator speed and small offset subtracted from the nominal generator speed (to avoid the control modes are switching all the time). In both control modes, the generator torque reference and the pitch reference $T_{g, r}$ and $\beta_{r}$ are the control parameters and the same reference is for all three pitch systems. 


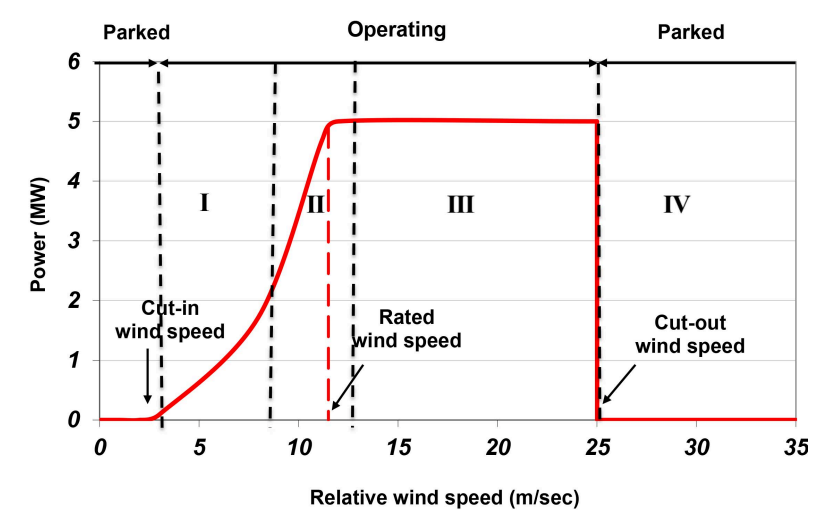

Figure 5. Illustration of the reference power curve for the wind turbine depending on the wind speed.

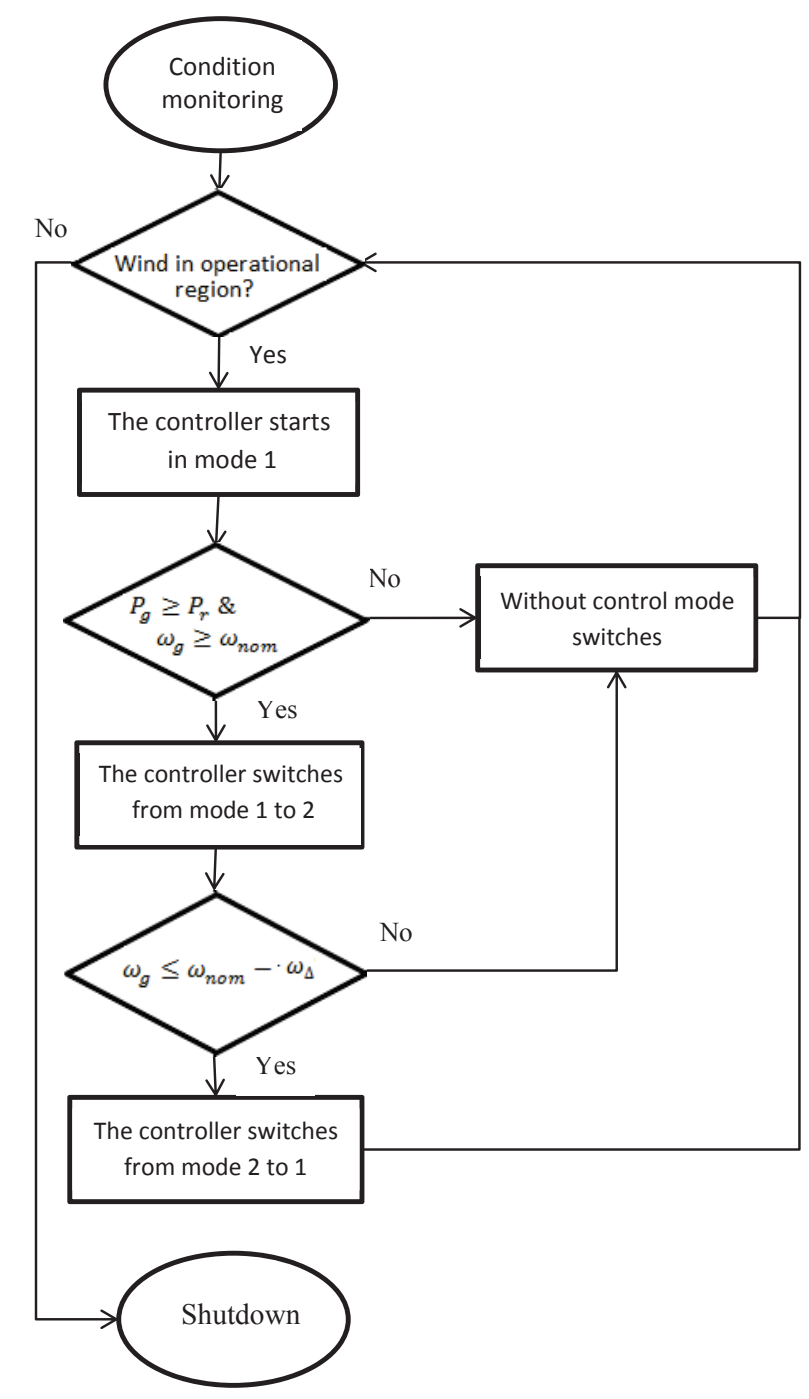

Figure 6. Control procedure for a wind turbine.

In control mode 1 , The optimal value of $\lambda$ is denoted $\lambda_{\text {opt }}$ is the optimum point in the torque coefficient $C_{p}$. This optimal value is achieved by setting the pitch reference to zero $\beta_{r}=0$ and the reference torque to the converter $T_{g, r}$ as follows:

$$
T_{g, r}=K_{o p t}\left(\frac{\omega_{g}}{N_{g}}\right)^{2}
$$

where

$$
K_{o p t}=\frac{1}{2} \rho A R^{3} \frac{C_{p_{\max }}}{\lambda_{o} p t^{3}}
$$

with $\rho$ the air density, $A$ the area swept by the turbine blades, and $C_{p_{\max }}$ the maximum value of the torque coefficient.

In control mode 2 , the pitch system using a PI controller trying to keep $\omega_{g}$ at $\omega_{\text {nom }}$ has been controlled.

$$
\dot{\beta}_{r}=K_{p} e+K_{i} \int e
$$

where $e=\omega_{g}-\omega_{n o m}$. In this case, the converter reference is used to suppress fast disturbances by

$$
T_{g, r}=\frac{P_{r}}{\eta_{g c} \omega_{g}}
$$

The used controller parameters can be found in Table 4 .

Table 4. Controller parameters used in the benchmark model Odgaard et al. (2009)

$$
\begin{array}{ll}
\omega_{\text {nom }}=162 \mathrm{rad} / \mathrm{s} & K_{\text {opt }}=1.2171 \\
\omega_{\delta}=15 \mathrm{rad} / \mathrm{s} & K_{i}=1 \\
P_{r}=4.8 \times 10^{6} \mathrm{~W} & K_{p}=4 \\
\hline
\end{array}
$$

A series of simulation results are presented to investigate the performance of the standard condition of the closed-loop system. Rotor and generator speed simulation responses are shown in Figure 7 for drivetrain and pitch positions in Figure 8 are shown for pitch subsystems. Figure 9 presents generator torque time series and its reference. Figure 10 presents power generated from the generator subsystem and power generated reference is $4.8 \mathrm{MW}$. The operating conditions are normal, and the system has been capable to meet its control objectives, the pitch angels and the generator torque. This is absolutely seen in the Figures 8, 9 .

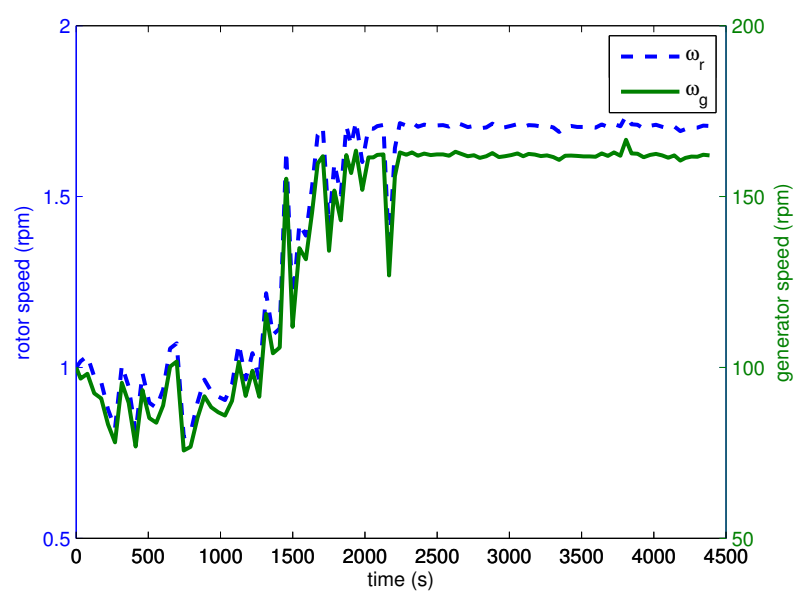

Figure 7. Rotor speed (blue dashed line) and generator speed (green solid line) time series for drivetrain subsystem.

\section{Fault scenario 1: faults in the drivetrain subsystem}

In this section the effectiveness of the proposed fault estimation design is demonstrated by applying it to a wind 


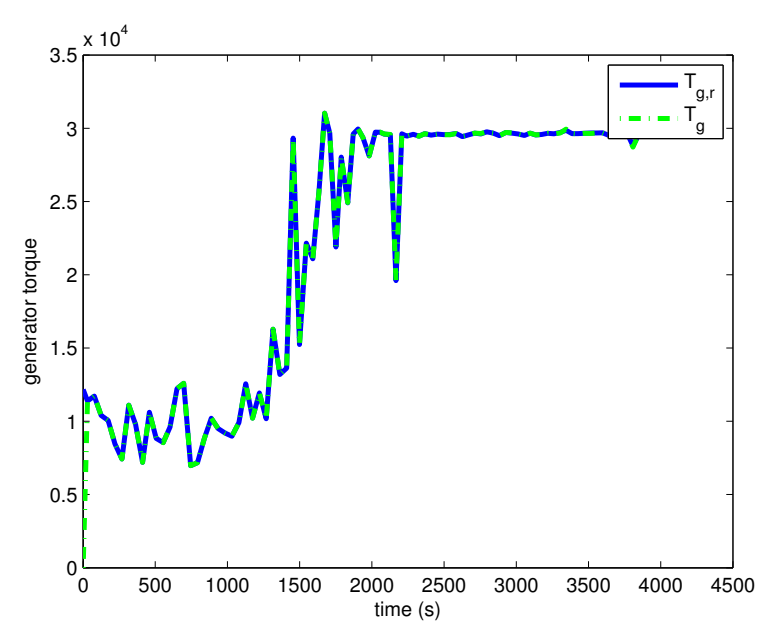

Figure 8. Generator torque reference (blue solid line) and generated by generator and converter subsystem (green dot-dashed line) time series.

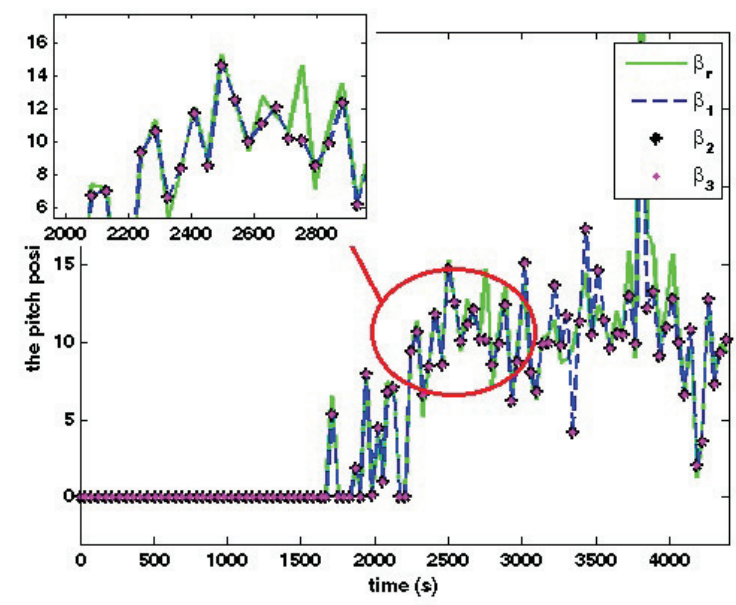

Figure 9. The pitch position (blue dashed line, black asterisk and magenta point) and its reference (green solid line) for blade 1,2 and 3 .

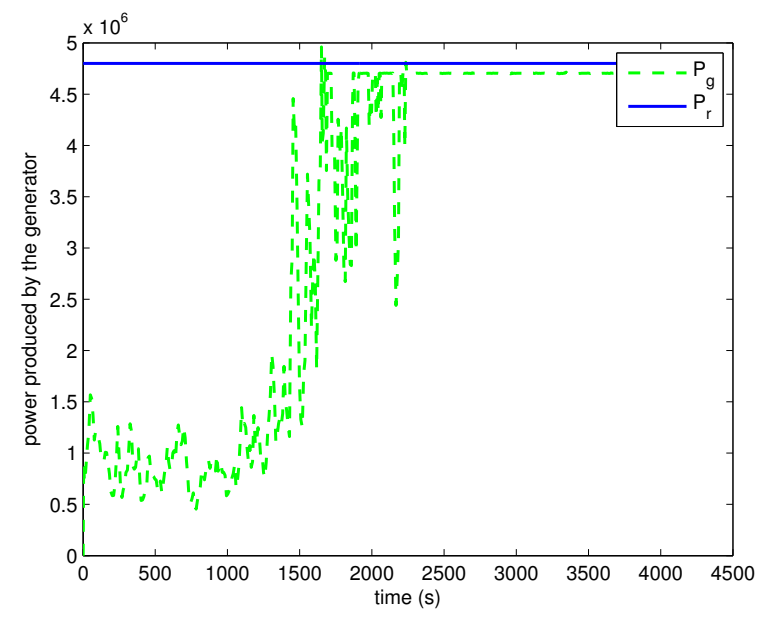

Figure 10. The power generated from the generator (green dashed line) and power refer to the wind turbine (blue solid line).

turbine benchmark Odgaard et al. (2009). The generator and rotor speed is simulated with a constant and ramp bias fault during $200 s-400 s, 1200 s-1400 s, 2200 s-2400 s$ and $3200 s-3400 s$. Step or ramp signals are the usual test signal that has used in this paper as the system is capable of responding and estimating different types of fault with the abrupt or incipient profile. The proposed method in this paper is not dependent to signal domain or time occurrence. The simulation results are shown in Figures 11-14.

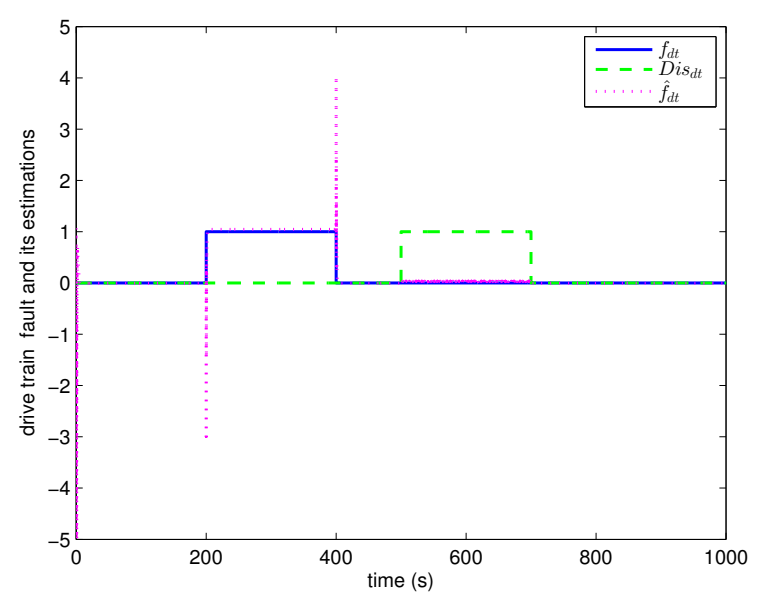

Figure 11. The drivetrain fault (blue solid line), disturbance (green dashed line) and fault estimated (magenta dot line) $\hat{f}$ in $0 s-1000 s$.

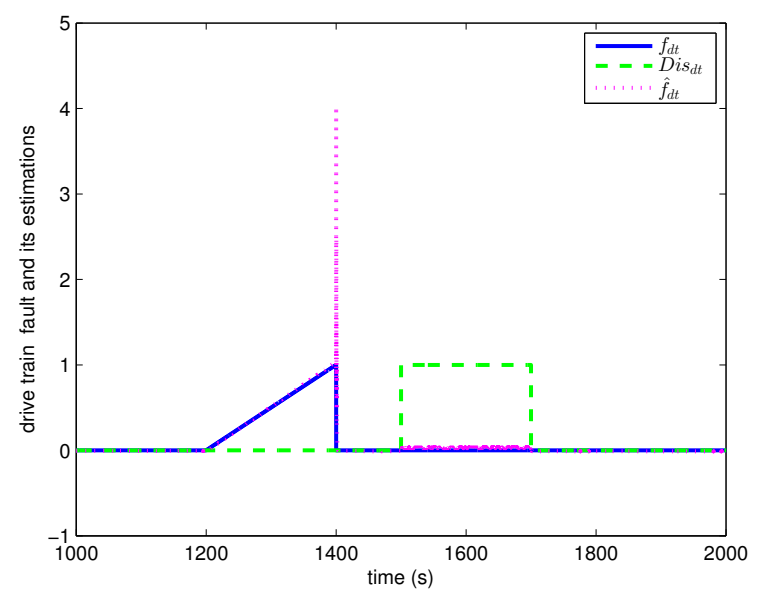

Figure 12. The drivetrain fault (blue solid line), disturbance (green dashed line) and fault estimated (magenta dot line) $\hat{f}$ in $1000 s-2000 s$.

where it can be seen that the estimated faults follows the real fault signals closely whether or not a disturbance has occurred. Distribution matrices are:

$$
E_{d}=\left[\begin{array}{l}
1 \\
0 \\
0
\end{array}\right], E_{c}=\left[\begin{array}{l}
0 \\
1 \\
0
\end{array}\right], F_{d}=\left[\begin{array}{l}
1 \\
1 \\
0
\end{array}\right], F_{c}=\left[\begin{array}{l}
0 \\
1 \\
0
\end{array}\right]
$$

and transformation matrices:

$$
T=\left[\begin{array}{lll}
1 & 0 & 0 \\
0 & 1 & 0 \\
0 & 0 & 1
\end{array}\right] \quad S=\left[\begin{array}{lcl}
-1.4142 & 0 & 0 \\
-0.7071 & 0.7071 & 0 \\
0 & 0 & 1
\end{array}\right]
$$




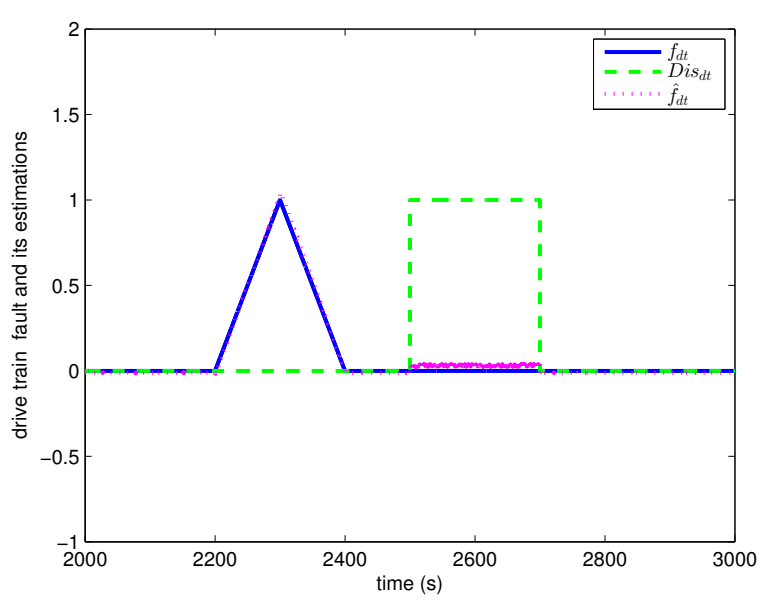

Figure 13. The drivetrain fault (blue solid line), disturbance (green dashed line) and fault estimated (magenta dot line) $\hat{f}$ in $2000 s-3000 s$.

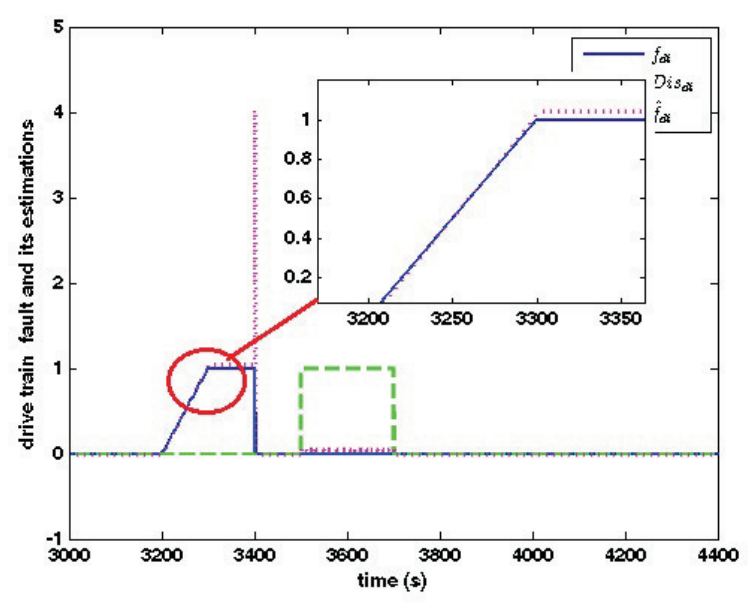

Figure 14. The drivetrain fault (blue solid line), disturbance (green dashed line) and fault estimated (magenta dot line) $\hat{f}$ in $3000 s-4400 s$.

The observer parameters are designed as follows:

RUIO: The RUIOs are proposed to disturbance and faults rejection separately. The observer parameters are selected as follow:

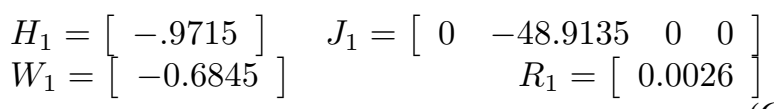

$$
\begin{aligned}
& \begin{array}{l}
H_{2}=\left[\begin{array}{ll}
-.0408 & 0.00151 \\
0 & -1.7169
\end{array}\right] \\
J_{2}=\left[\begin{array}{llr}
0.0042 & 0 & -0.0026 \\
0 & 0 & 0
\end{array}\right]
\end{array} \\
& W_{2}=\left[\begin{array}{lr}
1.4152 & 7.0685 e 4 \\
0 & 0
\end{array}\right] \\
& R_{2}=\left[\begin{array}{lr}
0.0001 & 0.0042 \\
0 & -1
\end{array}\right]
\end{aligned}
$$

RSMO: The RSMO scheme is designed to generate estimated faults in the pitch subsystem. The observer parameters are selected as follow:

$$
N=\left[\begin{array}{lr}
0.9600 & 7 e 4 \\
-0.0105 & 2
\end{array}\right] \quad k(.)=[2]
$$

The estimated fault is shown in Figures 11 - 14 in different duration, from which it is straightforward to determine whether there is a fault or not and also the severity of the fault. From the simulation results, there is an exact fault estimation by using the RUIO and RSMO scheme. There is different shape and domain for disturbance and fault signal, but the fault estimator could detect the fault and reject disturbance as early as possible. The high precision in tracking fault signals occurred at different operating times of the system indicates the effectiveness of the system. In fault signals with an abrupt change, the estimated signal has overshoot in their transient responses (Figure 11). But in incipient fault signals, there is no trace of overshoot (Figure 14). Although overshoot in transient response could not affect the estimator and it has followed the changes in the fault signals. The rapid reaction in this scheme shown in all figures will be effective for starting each FTC scheme. Another scenario that the fault and disturbance occurred in different duration is carried out in the following section.

\section{Fault scenario 2: faults in the pitch subsystem}

Faults in the pitch system influence the structural dynamics of the WT. Distribution matrices are:

$$
E_{d}=\left[\begin{array}{l}
0 \\
0 \\
1 \\
0 \\
0 \\
0
\end{array}\right], E_{c}=\left[\begin{array}{l}
1 \\
0 \\
0 \\
0 \\
1 \\
0
\end{array}\right], F_{d}=\left[\begin{array}{l}
0 \\
0 \\
1
\end{array}\right], F_{c}=\left[\begin{array}{l}
1 \\
1 \\
0
\end{array}\right]
$$

and transformation matrices:

$$
T=\left[\begin{array}{llclll}
0 & 0 & -1 & 0 & 0 & 0 \\
0 & 1 & 0 & 0 & 0 & 0 \\
-1 & 0 & 0 & 0 & 0 & 0 \\
0 & 0 & 0 & 1 & 0 & 0 \\
0 & 0 & 0 & 0 & 1 & 0 \\
0 & 0 & 0 & 0 & 0 & 1
\end{array}\right]
$$

Faults in actuators and sensors can be estimated effectively by RSMO. More information about observers schemes are listed as:

RUIO:

$$
\begin{aligned}
& H_{1}=[-5.1570] \\
& J_{1}=\left[\begin{array}{llllll}
0 & 0 & 32.8329 & 0 & 0 & -1
\end{array}\right] \\
& W_{1}=[1.0083] \\
& R_{1}=[0.0016]
\end{aligned}
$$




$$
\begin{aligned}
& H_{2}=\left[\begin{array}{lcc}
-123.5012 & -1.0006 & -58.0869 \\
246.7908 & -13.3325 & 99.7430 \\
0 & 0 & -0.6481 \\
-19.3941 & -0.1557 & 12.6707 \\
-115.8723 & 0.0606 & -55.1028
\end{array}\right. \\
& \left.\begin{array}{cc}
0 & 0 \\
0 & 0 \\
0 & 0 \\
-6.1560 & -11.6964 \\
1 & 0
\end{array}\right] \\
& J_{2}=\left[\begin{array}{rr}
0 & 0 \\
0 & -1 \\
0 & 0 \\
0 & -1 \\
0 & 0
\end{array}\right] \\
& W_{2}=\left[\begin{array}{rr}
0 & -1.0011 \\
0 & 1.0005 \\
0 & 0 \\
0 & -0.1592 \\
0 & -0.9380
\end{array}\right] \\
& R_{2}=\left[\begin{array}{lr}
0 & 0 \\
0 & 0 \\
-0.0305 & 0 \\
0 & -0.0013 \\
0 & 0.0005
\end{array}\right]
\end{aligned}
$$

\section{RSMO:}

$$
\begin{aligned}
& N=\left[\begin{array}{lcccr}
0.1 & -1 & 0 & 0 & 0 \\
123.4321 & -13.1320 & 0 & 0 & 0 \\
0 & 0 & 0.3 & 0 & 0 \\
0 & 0 & 0 & -5.656 & -11.6964 \\
0 & 0 & 0 & 1 & 0.15
\end{array}\right] \\
& k(.)=[20]
\end{aligned}
$$

The pitch subsystem fault scenarios are presented in terms of pitch angle variations in Figures 15-16. It is easy to know whether or not a fault has occurred from the provided estimation results. For example in Figure 15, it is clear that a pitch sensor fault occurred during $200-400$ has been estimated by the proposed fault estimation scheme carefully. Moreover, the estimated signal is not affected by the disturbance during $500-700$. The estimation of the fault signal $\hat{f}_{a}$ defined according to (54), is shown in Figures 1516. With the estimated fault signal, RSMO is carried out with the strategy presented in "Fault estimation scheme" section.

Faults in actuators and sensors can be detected effectively by the fault estimator scheme. Figure 15 shows the effects of a fault and disturbance in control mode. The fault has been estimated, and the disturbance has been rejected accurately. Figures 15 - 16 show effects the incipient fault in control mode 2. The results show that faults will be estimated accurately, and it is not dependent on the operating point of the wind turbine. This section and previous section provide some comparative results with respect to fault estimation scheme with a fault in the process and measurement with disturbance in the process and measurement of a wind turbine model. It was assumed that the process under investigation could be non-linear and its available measurements were usually not absolutely reliable, due to the wind speed uncertain nature. The simulation results are

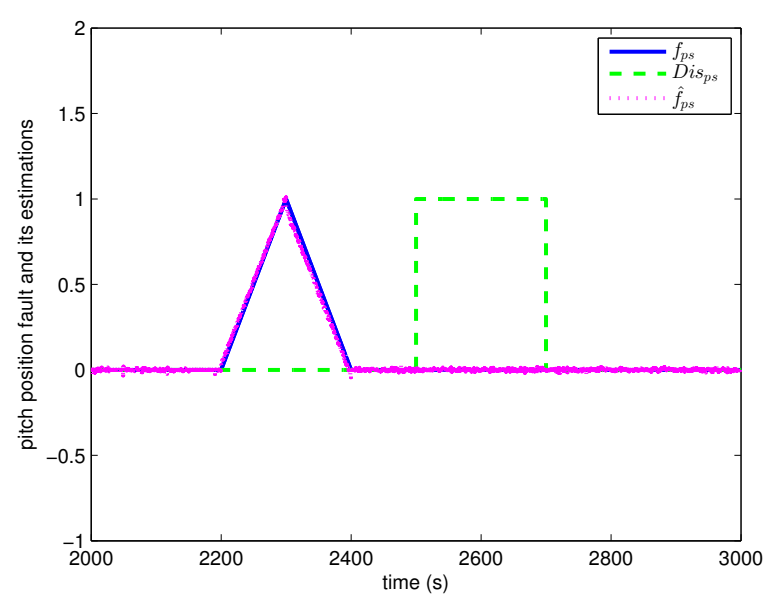

Figure 15. The pitch position fault (blue solid line), disturbance (green dashed line) and fault estimated (magenta dot line) $\hat{f}$ in $2000 s-3000 s$.

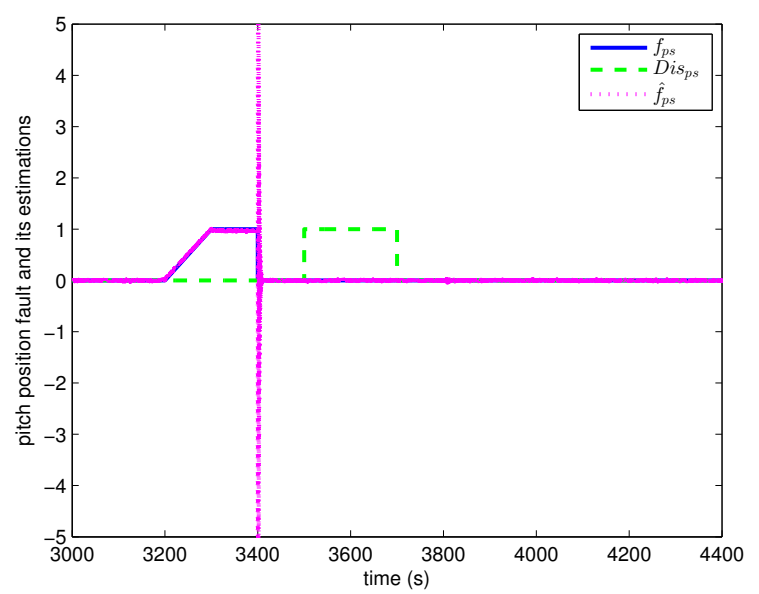

Figure 16. The pitch position fault (blue solid line), disturbance (green dashed line) and fault estimated (magenta dot line) $\hat{f}$ in $3000 s-4400 s$.

completely accurate for estimated fault and without any delay time that is shown in Figures 15 - 16 and also in Figures $11-14$.

\section{Conclusion and Future work}

The wind power generation systems have great potential to overcome the environmental problem of reducing carbon emissions. WTs are complex that depends on many factors on the cost associated with maintenance thus it is important to continue operating in the presence of faults, minimizing downtime and maximizing productivity. Each WT is completely different with foundation properties as well as environmental conditions like wind turbulence and waves. This paper has presented on a benchmark onshore version of a large WT for the simulations and analyzed to examine the design of the fault estimation scheme. At first, faults in pitch and drivetrain systems and disturbances in dynamic model and measurements are considered. Then, with proposed orthogonal transformations, faulty subsystems are separated from the rest of the system. Two reduced order unknown 
input and sliding mode observers designed to estimate sensor and actuators faults. Finally, sensor and actuators corrected with estimated faults. The novelty is the strategy is not depended to the current controller, but the result will be effective on the quality of the closed-loop system. This concept can also be applied in the different type of WTs with a variety of control methodologies where describes the complexity of the system, reference trajectories, memory capabilities and other different design factors. This strategy can be easily implemented in practice because of low data storage and off-line systematic mathematical operations. This method can easily expand to non-linear modelling of the physical systems. The system behaviours in simulations in healthy and faulty conditions are acceptable or close together. The proposed fault estimation scheme detects in a short time the shape and magnitude of the faults. This feature is a novelty for development of other fault tolerant schemes for WTs itself. The new scheme tracked the fault signal in the presence of any disturbance and rejected it totally, but SMO scheme could not achieve this. To compare old methodology, the effectiveness of this scheme is completely is shown by simulation results. Indeed, a robust scheme is proposed in order to be sensitive to a fault and insensitive to the disturbance or any unknown input signals. The most significant contribution of this article is to propose a novel and powerful scheme to remove disturbance in measurements and estimate sensor and component faults. The state observer, obtained via a Lyapunov function, and all the procedure is formulated by LMIs. The results of the application of these observers to sample models show satisfactory identification properties in the existence of output and input disturbances. We remark that the resulting strategies in this paper can be easily implemented in the realtime plant.

Some highlighted issue during the development of this paper has been detected that could be investigated in future work. The first is a different type of WTs structures, especially types of floating can be used to analyze the different environmental factors that belong to the systems. The second is the different types of faults. In this paper, faults in two subsystems of a WT were considered, but there are many sources of faults in present and also the future. The third is fault accommodation that has some limitation in compensation of process faults. Although the proposed fault estimation can conquer all types of faults, the control strategy for fault accommodation is not suitable for component types of faults.

\section{References}

Aldeen M and Sharma R (2008) Estimation of states, faults and unknown disturbances in non-linear systems. International Journal of Control 81(8): 1195-1201.

Alessandri A (2004) Observer design for nonlinear systems by using input-to-state stability. In: Decision and Control, 2004. CDC. 43rd IEEE Conference on, volume 4. IEEE, pp. 38923897.

Azizi A, Nourisola H and Shoja-Majidabad S (2019) Fault tolerant control of wind turbines with an adaptive output feedback sliding mode controller. Renewable Energy 135: 55-65.
Azmi H and Khosrowjerdi MJ (2016) Robust adaptive fault tolerant control for a class of lipschitz nonlinear systems with actuator failure and disturbances. Proceedings of the Institution of Mechanical Engineers, Part I: Journal of Systems and Control Engineering 230(1): 13-22.

Chen J and Patton RJ (2012) Robust model-based fault diagnosis for dynamic systems, volume 3. Springer Science \& Business Media.

Chen W, Ding SX, Haghani A, Naik A, Khan AQ and Yin S (2011) Observer-based fdi schemes for wind turbine benchmark. IFAC Proceedings Volumes 44(1): 7073-7078.

Cho S, Gao Z and Moan T (2018) Model-based fault detection, fault isolation and fault-tolerant control of a blade pitch system in floating wind turbines. Renewable Energy 120: 306-321.

Dong J and Verhaegen M (2011) Data driven fault detection and isolation of a wind turbine benchmark. IFAC Proceedings Volumes 44(1): 7086-7091.

Edwards C and Spurgeon S (1998) Sliding mode control: theory and applications. CRC Press.

Feng Z and Liang M (2014) Fault diagnosis of wind turbine planetary gearbox under nonstationary conditions via adaptive optimal kernel time-frequency analysis. Renewable Energy 66: 468-477.

Fu J, Chu J, Guo P and Chen Z (2019) Condition monitoring of wind turbine gearbox bearing based on deep learning model. IEEE Access 7: 57078-57087.

Gao Z, Liu X and Chen MZ (2016) Unknown input observerbased robust fault estimation for systems corrupted by partially decoupled disturbances. IEEE Trans. Industrial Electronics 63(4): 2537-2547.

Ghane M, Nejad AR, Blanke M, Gao Z and Moan T (2016) Statistical fault diagnosis of wind turbine drive train applied to a 5mw floating wind turbine. In: Journal of Physics: Conference Series, volume 753. IOP Publishing, p. 052017.

Ghaniee M and Shoorehdeli MA (2011) Fault detection of nonlinear systems by parity relations. In: The 2nd International Conference on Control, Instrumentation and Automation. IEEE, pp. 519-523.

Gillijns S and De Moor B (2007) Unbiased minimum-variance input and state estimation for linear discrete-time systems. Automatica 43(1): 111-116.

Guo R, Guo K, Dong J and Zhu Y (2015) Time-varying and antidisturbance fault diagnosis for a class of nonlinear systems. Proceedings of the Institution of Mechanical Engineers, Part I: Journal of Systems and Control Engineering 229(7): 573-586.

Hassan KK (2002) Nonlinear systems. Prentice Hall, Inc.

Huang Q, Jiang D, Hong L and Ding Y (2008) Application of wavelet neural networks on vibration fault diagnosis for wind turbine gearbox. In: International Symposium on Neural Networks. Springer, pp. 313-320.

Jonkman JM, Buhl Jr ML et al. (2005) Fast user's guide. National Renewable Energy Laboratory, Golden, CO, Technical Report No. NREL/EL-500-38230 .

Ke Zhang BJ and Cocquempot V (2016) Fuzzy unknown input observer-based robust fault estimation design for discrete-time fuzzy systems. Signal Processing 128: 40 - 47.

Lan J and Patton RJ (2017) Integrated fault estimation and faulttolerant control for uncertain lipschitz nonlinear systems. International Journal of Robust and Nonlinear Control 27(5): 
761-780

Laouti N, Sheibat-Othman N and Othman S (2011) Support vector machines for fault detection in wind turbines. IFAC Proceedings Volumes 44(1): 7067-7072.

Liu X, Gao Z and Chen MZ (2017) Takagi-sugeno fuzzy model based fault estimation and signal compensation with application to wind turbines. IEEE Transactions on Industrial Electronics 64(7): 5678-5689.

Malik H and Mishra S (2015) Application of probabilistic neural network in fault diagnosis of wind turbine using fast, turbsim and simulink. Procedia Computer Science 58: 186-193.

Marquez FPG, Tobias AM, Perez JMP and Papaelias M (2012) Condition monitoring of wind turbines: Techniques and methods. Renewable Energy 46: 169-178.

Mellucci C, Menon PP, Edwards C and Ferrara A (2017) Secondorder sliding mode observers for fault reconstruction in power networks. IET Control Theory \& Applications 11(16): 27722782.

Nazir M, Khan AQ, Mustafa G and Abid M (2017) Robust fault detection for wind turbines using reference model-based approach. Journal of King Saud University-Engineering Sciences 29(3): 244-252.

Odgaard PF, Stoustrup J and Kinnaert M (2009) Fault tolerant control of wind turbines-a benchmark model. IFAC Proceedings Volumes 42(8): 155-160.

Progovac D, Wang LY and Yin G (2014) Parameter estimation and reliable fault detection of electric motors. Control Theory and Technology 12(2): 110-121.

Qiao W and Lu D (2015) A survey on wind turbine condition monitoring and fault diagnosis-part i: Components and subsystems. IEEE Transactions on Industrial Electronics 62(10): 6536-6545.

Raoufi R (2010) Nonlinear robust observers for simultaneous state and fault estimation .

Raoufi R, Marquez HJ and Zinober ASI (2010) $h_{\text {inf }}$ sliding mode observers for uncertain nonlinear lipschitz systems with fault estimation synthesis. International Journal of Robust and Nonlinear Control 20(16): 1785-1801.

Raoufi R and Marquezz H (2010) Simultaneous sensor and actuator fault reconstruction and diagnosis using generalized sliding mode observers. In: American Control Conference (ACC). IEEE, pp. 7016-7021.

Sahin S, Tolun MR and Hassanpour R (2012) Hybrid expert systems: A survey of current approaches and applications. Expert systems with applications 39(4): 4609-4617.

Saoudi D, Chadli M and Braeik NB (2015) Robust estimation design for unknown inputs fuzzy bilinear models: Application to faults diagnosis. In: Complex System Modelling and Control Through Intelligent Soft Computations. Springer, pp. 655-685.

Schlechtingen M and Santos IF (2011) Comparative analysis of neural network and regression based condition monitoring approaches for wind turbine fault detection. Mechanical systems and signal processing 25(5): 1849-1875.

Simani S and Castaldi P (2019) Intelligent fault diagnosis techniques applied to an offshore wind turbine system. Applied Sciences 9(4): 783.

Tan CP and Edwards C (2003) Sliding mode observers for robust detection and reconstruction of actuator and sensor faults. International Journal of Robust and Nonlinear Control 13(5):
443-463.

Valibeygi A, Toudeshki A and Vijayaraghavan K (2016) Observer-based sensor fault estimation in nonlinear systems. Proceedings of the Institution of Mechanical Engineers, Part I: Journal of Systems and Control Engineering 230(8): 759-777.

Veluvolu KC, Kim M and Lee D (2011) Nonlinear sliding mode high-gain observers for fault estimation. International Journal of Systems Science 42(7): 1065-1074.

Wang L, Zhang Z, Xu J and Liu R (2016) Wind turbine blade breakage monitoring with deep autoencoders. IEEE Transactions on Smart Grid 9(4): 2824-2833.

Wang T, Han Q, Chu F and Feng Z (2019) Vibration based condition monitoring and fault diagnosis of wind turbine planetary gearbox: A review. Mechanical Systems and Signal Processing 126: 662-685.

Wei X, Verhaegen M and van Engelen T (2010) Sensor fault detection and isolation for wind turbines based on subspace identification and kalman filter techniques. International Journal of Adaptive Control and Signal Processing 24(8): 687707.

Witczak M (2014) Unknown input observers and filters. In: Fault Diagnosis and Fault-Tolerant Control Strategies for Non-Linear Systems, Lecture Notes in Electrical Engineering, volume 266. Springer International Publishing, pp. 19-56.

Witczak M, Buciakowski M, Puig V, Rotondo D and Nejjari F (2016) An lmi approach to robust fault estimation for a class of nonlinear systems. International Journal of Robust and Nonlinear Control 26(7): 1530-1548.

Wu X, Jiang G, Wang X, Xie P and Li X (2019) A multilevel-denoising autoencoder approach for wind turbine fault detection. IEEE Access 7: 59376-59387.

Yan XG and Edwards C (2007) Nonlinear robust fault reconstruction and estimation using a sliding mode observer. Automatica 43(9): 1605-1614.

Yin S, Yang X and Karimi HR (2012) Data-driven adaptive observer for fault diagnosis. Mathematical Problems in Engineering 2012.

Zhang $\mathrm{Y}$ and Jiang $\mathrm{J}$ (2008) Bibliographical review on reconfigurable fault-tolerant control systems. Annual reviews in control 32(2): 229-252.

Zhang Z, Verma A and Kusiak A (2012) Fault analysis and condition monitoring of the wind turbine gearbox. IEEE transactions on energy conversion 27(2): 526-535.

Ziyabari SHS and Shoorehdeli MA (2017a) Robust fault diagnosis scheme in a class of nonlinear system based on uio and fuzzy residual. International Journal of Control, Automation and Systems 15(3): 1145-1154.

Ziyabari SHS and Shoorehdeli MA (2017b) Robust fuzzy fault estimation based on decoupled transform and unknown input sliding mode observer. In: Electrical Engineering (ICEE), 2017 Iranian Conference on. IEEE, pp. 772-777.

Ziyabari SHS and Shoorehdeli MA (2018) Fuzzy robust fault estimation scheme for a class of nonlinear systems based on an unknown input sliding mode observer. Journal of Vibration and Control 24(10): 1861-1873. 